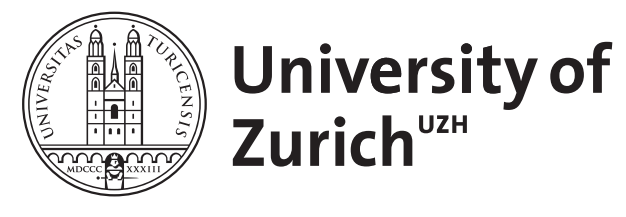

\title{
Normalisation holomorphe de structures de Poisson
}

\author{
Lohrmann, Philipp
}

\begin{abstract}
Nous montrons qu'une structure de Poisson à 1-jet nul est holomorphiquement conjuguée vers une forme normale au sens de Dufour-Wade, au voisinage de son point singulier $0 \in \mathbb{C}^{n}$, si sont vérifiées d'une part une condition diophantienne sur une algèbre de Lie associée à la partie quadratique, d'autre part certaines conditions sur la forme normale formelle. We show that a Poisson structure whose linear part vanishes can be holomorphically normalized in a neighbourhood of its singular point $0 \in \mathbb{C}^{n}$ if, on the one hand, a Diophantine condition on a Lie algebra associated to the quadratic part is satisfied and, on the other hand, the normal form satisfies some formal conditions.
\end{abstract}

DOI: https://doi.org/10.1017/s0143385709000480

Posted at the Zurich Open Repository and Archive, University of Zurich ZORA URL: https://doi.org/10.5167/uzh-154013

Journal Article

Published Version

Originally published at:

Lohrmann, Philipp (2010). Normalisation holomorphe de structures de Poisson. Ergodic Theory and Dynamical Systems, 30(4):1165-1199.

DOI: https://doi.org/10.1017/s0143385709000480 


\title{
Normalisation holomorphe de structures de Poisson
}

\author{
PHILIPP LOHRMANN \\ Institut für Mathematik, Universität Zürich, Winterthurerstrasse 190, 8057 Zürich, \\ Switzerland \\ (e-mail: philipp.lohrmann@math.uzh.ch)
}

(Received 9 September 2008 and accepted in revised form 24 March 2009)

Résumé. Nous montrons qu'une structure de Poisson à 1-jet nul est holomorphiquement conjuguée vers une forme normale au sens de Dufour-Wade, au voisinage de son point singulier $0 \in \mathbb{C}^{n}$, si sont vérifiées d'une part une condition diophantienne sur une algèbre de Lie associée à la partie quadratique, d'autre part certaines conditions sur la forme normale formelle.

Abstract. We show that a Poisson structure whose linear part vanishes can be holomorphically normalized in a neighbourhood of its singular point $0 \in \mathbb{C}^{n}$ if, on the one hand, a Diophantine condition on a Lie algebra associated to the quadratic part is satisfied and, on the other hand, the normal form satisfies some formal conditions.

\section{Introduction}

Soient $M$ une variété analytique de dimension $n, p \in M$ et $C^{\omega}$ l'anneau des germes en $p$ de fonctions holomorphes sur $M$. Une structure de Poisson en $p$ est la donnée d'une loi de composition interne, $\{\cdot, \cdot\}: C^{\omega} \times C^{\omega} \rightarrow C^{\omega}$, appelée crochet de Poisson. Cette loi est bilinéaire, antisymétrique et telle que pour tout $f, g, h \in C^{\omega}$ :

(i) les applications $\{f, \cdot\}$ et $\{\cdot, f\}$ soient des dérivations de l'anneau $C^{\omega}$;

(ii) on ait la relation $\{f,\{g, h\}\}+\{g,\{h, f\}\}+\{h,\{f, g\}\}=0$ (identité de Jacobi).

De manière équivalente, on peut, au voisinage de $p$, se donner un champ de bivecteurs $\Pi$, de la forme $\Pi(x)=\sum_{i<j} \Pi_{i j}(x) \partial_{i} \wedge \partial_{j}, \partial_{i} \equiv \partial / \partial x_{i}$, en coordonnées locales. Le crochet associé est alors défini par $\left\{x_{i}, x_{j}\right\}:=\Pi_{i j}$, i.e. $\{f, g\}=\sum_{i<j} \Pi_{i j}\left(\partial_{i} f \partial_{j} g-\partial_{i} g \partial_{j} f\right)$, et l'identité de Jacobi équivaut à ce que pour tout $i, j, k$ on ait

$$
\sum_{l=1}^{n} \Pi_{i l} \partial_{l} \Pi_{j k}+\Pi_{j l} \partial_{l} \Pi_{k i}+\Pi_{k l} \partial_{l} \Pi_{i j}=0 .
$$


Soit $f \in C^{\omega}$. On note $X_{\Pi}(f)$ le champ de vecteurs hamiltonien de $f$ relativement à $\Pi$, tel que pour tout $g \in C^{\omega}$, on ait $\mathcal{L}_{X_{\Pi}(f)}(g)=\{f, g\}$, soit

$$
X_{\Pi}(f)=\sum_{i<j} \Pi_{i j}\left(\partial_{i} f \partial_{j}-\partial_{j} f \partial_{i}\right)
$$

en coordonnées locales. On dit que $f \in C^{\omega}$ est une fonction de Casimir si on a $\{f, g\}=0$ pour tout $g \in C^{\omega}$. S. Lie a montré qu'au voisinage d'un point $p \in M$ où le rang de $\Pi$ reste constant, on peut décomposer $M$ en un produit direct $M=\mathcal{S} \otimes \mathcal{N}$ d'une variété symplectique $\mathcal{S}$ et d'une variété complémentaire $\mathcal{N}$ sur laquelle tous les crochets sont nuls $[\mathbf{1 0}]$.

Dans la catégorie holomorphe, l'étude locale des structures de Poisson se limite aux points singuliers, c'est-à-dire des point où elles s'annulent. A. Weinstein $[\mathbf{1 0}]$ a en effet montré au début des années 80 le résultat suivant: soit $\Pi$ une structure de Poisson sur une variété holomorphe $M$, et soit $p \in M$ tel que $\Pi(p) \neq 0$. Il existe alors en $p$ des coordonnées locales holomorphes $\left(p_{1}, \ldots, p_{r}, q_{1}, \ldots, q_{r}, x_{1}, \ldots, x_{s}\right), r, s \in \mathbb{N}$, $n=2 r+s$, telles que

$$
\Pi=\sum_{1 \leq i<j \leq r} \partial / \partial p_{i} \wedge \partial / \partial q_{i}+\sum_{1 \leq i<j \leq s} \pi_{i j}(x) \partial / \partial x_{i} \wedge \partial / \partial x_{j}, \quad x=\left(x_{1}, \ldots, x_{s}\right),
$$

avec $\pi_{i j}(0)=0$. Sous l'impulsion de Weinstein, on s'est d'abord intéressé au problème de la linéarisation, c'est-à-dire la conjuguaison d'une structure de Poisson à sa partie linéaire par le moyen d'une transformation biholomorphe au voisinage de $0 \in \mathbb{C}^{n}$. Pour ce problème, le résultat de J. Conn (voir [3]) est primordial: si l'algèbre de Lie associée sur le cotangent est semi-simple, alors la structure de Poisson est holomorphiquement linéarisable. On a aussi un résultat analogue dans la catégorie $C^{\infty}$ (voir [4]). Stolovitch, dans [9], a établi l'existence d'une conjuguaison holomorphe vers une forme normale pour certaines classes de structures de Poisson singulières admettant une partie linéaire, mais pas nécessairement formellement linéarisables.

Ensuite on s'est intéressé au cas où la partie linéaire s'annule, mais où la partie quadratique est non-nulle. L'intérêt pour ce problème vient d'une part du fait que ce cas de figure est structurellement stable (voir section 2). D'autre part, les structures de Poisson quadratiques jouent, via les r-matrices, un rôle très important dans les systèmes intégrables_-pour plus de détails et la Bibliographie on revoie le lecteur au livre récent [7]. Les premiers résultats ont été obtenus par V. I. Arnol'd, qui a étudié les structures de Poisson quadratiques en dimension 2 [1]. Dufour et Wade ont défini, dans [6] une forme normale formelle pour les structures de Poisson à 1 -jet nul qui est assez semblable de celle de Poincaré-Dulac pour les champs de vecteurs nuls en un point. Ils ont donné un résultat de quadratisation dans la catégorie $C^{\infty}$, c'est-à-dire la conjuguaison au 2-jet par le biais d'un germe de transformation $C^{\infty}$. Pour plus d'informations sur les structures de Poisson le lecteur pourra consulter les livres $[\mathbf{1 , 7}]$.

Dans cet article est établi pour les structures de Poisson analytiques à 1-jet nul en un point, (admettant une forme normale formelle de Dufour-Wade), un théorème à la BrunoStolovitch, c'est-à-dire de mise sous forme normale par le biais d'une transformation holomorphe sous deux conditions : 
(i) une condition algébrique sur la forme normale formelle ;

(ii) une condition de petits diviseurs diophantiens : on sait associer à la partie quadratique une suite de nombres réels, appelés petits diviseurs. On demande que ces nombres ne s'accumulent pas trop vite sur 0 .

On obtient comme corollaire, qu'une structure de Poisson formellement quadratisable l'est holomorphiquement sous une condition diophantienne. Cette condition diophantienne est très naturelle, et beaucoup plus faible que celle utilisée dans le résultat de quadratisation holomorphe de Dufour et Zung dans [7].

La condition algébrique sur la forme normale formelle qu' on utilise est intrinsèque : elle ne dépend pas de la forme normale (qui n'est pas unique). Sa propriété la plus importante est l'identité de l'ensemble des fonctions de Casimir relatives à la partie quadratique d'une part, et relatives à la forme normale d'autre part. Il suit une remarquable interprétation géométrique portant sur les variétés symplectiques passant par les points voisin du point singulier (cf. le résultat de Lie rappelé plus haut).

On utilisera une méthode de type Newton qui est devenue classsique et qui a été introduite par Kolmogorov et Arnol'd. La démonstration s'inspire des méthodes que Stolovitch, dans [8], a développé pour montrer son résultat sur la normalisation simultanée d'une famille abélienne de champs de vecteurs. Ce travail s'est fait dans le cadre d'une thèse sous la direction de L. Stolovitch, et l'auteur le remercie chalereusement pour son aide. L'auteur remercie également H. Eliasson, J.-P. Françoise et B. Malgrange, pour leurs suggestions qui ont permis d'améliorer la rédaction de cet article.

\section{Rappels et notations}

Dans cette section on rassemble des résultats sur les structures de Poisson et la forme normale de Dufour-Wade qui sont utilisés dans la suite. Le lecteur est invité à la consulter au fur et à mesure des besoins.

Définition 2.1. (Champ de bivecteurs quadratique diagonal) Un champ de bivecteurs quadratique $\Pi$ de la forme $\Pi=(1 / 2) \sum_{i \neq j} q_{i j} x_{i} x_{j} \partial_{i} \wedge \partial_{j}, q_{i j}=-q_{j i}$, est dit diagonal.

Remarque. (i) D'après [6], proposition 1, l'expression diagonale est unique, modulo permutation des lignes de la matrice $\left(q_{i j}\right)$.

(ii) Tout champ de bivecteurs quadratique diagonal définit une structure de Poisson.

Notation. Pour tout $1 \leq i \leq n$, on pose $Y_{i}:=x_{i} \partial_{i}$.

Généricité de structures de Poisson à 1-jet nul et à partie quadratique diagonalisable. Dufour et Wade [6], et Wade [11] ont obtenus les propriétés suivantes: soit $\Pi^{0}=$ $(1 / 4) \sum_{i, j, k, l} \Pi_{i j}^{k l} x_{k} x_{l} \partial_{i} \wedge \partial_{j}$ une structure de Poisson quadratique. On sait associer à $\Pi^{0}$ un champ de vecteurs $X$, appelé le rotationnnel, en posant $X:=\sum_{i, j, k} \Pi_{i j}^{k j} x_{k} \partial_{i}$. Si les valeurs propres de $X$ ne vérifient pas de relation du type $\lambda_{i}+\lambda_{j}=\lambda_{r}+\lambda_{s}$, où $(i, j) \neq(r, s)$, alors $\Pi^{0}$ est diagonalisable. De plus, si aucune relation $\lambda_{i}+\lambda_{j}=0$, $\lambda_{i}+\lambda_{j}=\lambda_{k}$ n'est satisfaite, une structure de Poisson $\Pi, C^{2}$-proche $\Pi^{0}$, possède son 1 -jet nul, en un point $p$ proche de 0 . 
Crochet de Schouten. Désignons par $\chi_{1}$ l'espace des germes de champs de vecteurs en 0 (respectivement champs de vecteurs formels), et par $\chi_{p}:=\bigwedge^{p} \chi_{1}$, l'espace des germes de champs de $p$-vecteurs. On définit (voir [7]) le crochet de Schouten-Nijenhuis $[\cdot, \cdot]: \chi_{p} \times \chi_{q} \rightarrow \chi_{p+q-1}, \mathbb{C}$-bilinéaire et extension de la dérivée de Lie, tel que

$$
\begin{gathered}
{[P, Q]=-(-1)^{(p-1)(q-1)}[Q, P]} \\
{[P, Q \wedge R]=[P, Q] \wedge R+(-1)^{(p-1) q} Q \wedge[P, R],} \\
(-1)^{(p-1)(r-1)}[P,[Q, R]]+(-1)^{(q-1)(p-1)}[Q,[R, P]] \\
+(-1)^{(r-1)(q-1)}[R,[P, Q]]=0 .
\end{gathered}
$$

Un champ de 2-vecteurs $\Pi$ définit une structure de Poisson, si et seulement si $[\Pi, \Pi]=0$. Le champ hamiltonien $X_{\Pi}(f)$ d'une fonction $f$ relativement à une structure de Poisson $\Pi$ est donné par

$$
X_{\Pi}(f)=[f, \Pi] .
$$

Soit $X$ un champ de vecteurs formel sur $M$ nul à l'ordre deux. On a alors la formule bien connue

$$
\exp (X)^{*}(B)=\sum_{i \geq 0} \frac{\operatorname{ad}_{X}^{i} B}{i !},
$$

où on a noté $\operatorname{ad}_{X}$ l'application linéaire $[X, \cdot]$, et $\exp (X)$ le temps 1 du flot formel du champ formel $X$. Pour plus d'informations sur le crochet de Schouten on renvoie le lecteur à [7].

Champs hamiltoniens. La notion de champ hamiltonien relativement à une structure de Poisson s'étend aux champ de bivecteurs.

Définition 2.2. Le champ hamiltonien $X_{P}(f)$ d'une fonction holomorphe $f$ relativement à un champ de bivecteurs $P$ est défini comme la contraction du 2-tenseur contravariant $P$ avec le 1-tenseur covariant $d f$.

Pour tous champs de bivecteurs $P$ et toute fonction holomorphe $f$ on a

$$
\mathcal{L}_{X_{P}(f)}(f)=0 .
$$

En effet, $\mathcal{L}_{X_{P}(f)}(f)$ est la contraction de $P$ avec $d f \wedge d f=0$.

Pour un multi-indice $I=\left(I_{1}, \ldots, I_{n}\right)$ et une structure de Poisson $\Pi:=(1 / 2)$ $\sum_{i \neq j} a_{i j} Y_{i} \wedge Y_{j}$ on a

$$
X_{\Pi}\left(x^{I}\right)=-x^{I} \sum_{i=1}^{n} \mathcal{L}_{A_{i}}\left(x^{I}\right) Y_{i}, \quad \text { avec } \quad A_{i}:=\sum_{j=1}^{n} a_{i j} Y_{j} .
$$

Soient $\Pi, \Pi^{\prime}$ des structures de Poissons quadratiques diagonales, $Z$ un champ de vecteurs linéaire diagonal et $J, K \in \mathbb{Z}^{n}$ des multi-indices. Par (2.1)-(2.4),

$$
\begin{gathered}
{\left[\Pi, x^{J}\right]=-X_{\Pi}\left(x^{J}\right),} \\
{\left[\Pi, x^{J} Z\right]=Z \wedge X_{\Pi}\left(x^{J}\right),} \\
{\left[x^{J} \Pi, x^{K} \Pi^{\prime}\right]=2\left(X_{\Pi^{\prime}}\left(x^{J}\right) \wedge \Pi+X_{\Pi}\left(x^{K}\right) \wedge \Pi^{\prime}\right) .}
\end{gathered}
$$

De (2.7)-(2.10) on déduit le lemme suivant. 
LEMME 2.1 .

(i) Soient $I, J \in \mathbb{Z}^{n}$ des multi-indices, $Z$ un champ de vecteurs linéaire diagonal, $\Pi$ une structure de Poisson quadratique diagonale. On a alors

$$
\left[x^{I} Z, x^{J} \Pi\right]=x^{I+J} \Pi^{\prime},
$$

où $\Pi^{\prime}$ est une structure de Poisson quadratique diagonale.

(ii) Soient $\Pi^{I}$ et $\Pi^{J}$ deux structures de Poisson quadratiques diagonaux. Alors $\left[x^{I} \Pi^{I}, x^{J} \Pi^{J}\right]$ s'écrit sous la forme

$$
x^{I+J} \sum_{1 \leq i<j<k \leq n} c_{i j k} Y_{i} \wedge Y_{j} \wedge Y_{k}, \quad c_{i j k} \in \mathbb{C} .
$$

Définition 2.3. Soit $\Pi$ une structure de Poisson. Une fonction $f$ est une fonction de Casimir si $X_{\Pi}(f)=0$, i.e. si $\{f, \cdot\}=0$.

Normes sur les polydisques. Soit $f:=\sum_{|I|>0} c^{I} x^{I}$ holomorphe sur le polydisque $|x| \leq r$. Posons $\|f\|_{r}:=\sum_{|I|>0}\left|c^{I}\right| r^{|I|}$. Pour un champ de vecteurs $F:=\left(f_{1}, \ldots, f_{n}\right)$, holomorphe sur le polydisque $|x| \leq r$, on pose $\|F\|_{r}:=\max _{1 \leq i \leq n}\left\|f_{i}\right\|_{r}$. Si

$$
\Pi:=\frac{1}{2} \sum_{i \neq j} a_{i j}(x) \partial_{i} \wedge \partial_{j}, \quad a_{i j}(x)=-a_{j i}(x),
$$

est un champ de bivecteurs holomorphe sur le polydisque $|x| \leq r$, on définit $\|\Pi\|_{r}:=$ $\max _{i \neq j}\left\|a_{i j}(x)\right\|_{r}$. Finalement, pour un champ d'endomorphismes $A$ de $\mathbb{C}^{n}$, holomorphe sur le polydisque $|x| \leq r$, c'est-à-dire un champ de matrices $\left(a_{i j}(x)\right)_{1 \leq i, j \leq n}$, notons $\|A\|_{r}:=\max _{1 \leq i, j \leq n}\left\|a_{i j}(x)\right\|_{r}$.

Forme normale de Dufour-Wade. Dorénavant on considère les structures de Poisson à 1-jet nul à l'origine, admettant une partie quadratique $\Pi^{0}$ diagonale. On note

$$
\Pi^{0}=\frac{1}{2} \sum_{i \neq j} a_{i j} Y_{i} \wedge Y_{j}, \quad a_{i j}=-a_{j i},
$$

l'écriture diagonale de $\Pi^{0}$. Dufour et Wade ont donné une notion de forme normale formelle pour les structures de Poisson à 1-jet nul qui est assez proche de celle des champs de vecteurs nuls en un point : la matrice $A:=\left(a_{i j}\right)$ jouant le rôle du spectre de la partie linéaire du champ de vecteurs. La lettre $I$ désigne des multi-indices appartenant à $(\mathbb{N} \cup\{-1\})^{n}$. L'hypothèse $(\mathrm{H})$ est une hypothèse générique portant sur la partie quadratique qui est précisé plus bas.

ThÉorème-DÉfinition. (Dufour-Wade) Toute structure de Poisson à 1-jet nul à l'origine et dont la partie quadratique, de forme (2.11), satisfait l'hypothèse (H) est formellement conjuguée à une structure de Poisson du type

$$
\Pi=\sum_{i<j} \sum x^{I} a_{i j}^{I} Y_{i} \wedge Y_{j}
$$

la seconde somme étant prise sur les multi-indices $I \in(\mathbb{N} \cap\{-1\})^{n}$ pour lesquels $x^{I}$ est une fonction de Casimir relativement à la partie quadratique $\Pi^{0}$. Une structure de Poisson formelle à 1-jet nul de forme (2.12) est dite sous forme normale au sens de Dufour-Wade. 
Remarque. Notons $S_{i}$ le champ de vecteurs diagonal $\sum_{j=1}^{n} a_{i j} Y_{j}$. Le monôme $x^{I}$ est une fonction de Casimir relativement à la partie quadratique $\Pi^{0}$ équivaut à ce que pour tout $1 \leq i \leq n$, on a $\mathcal{L}_{S_{i}} x^{I}=0$.

Définition 2.4. (Terme résonnant) On appelle terme résonnant, un terme de la forme du second membre de (2.12), c'est-à-dire un champ de bivecteurs polynomial s'écrivant sous la forme $x^{I} \Pi^{I}$, où le $n$-uplet $I$ appartient à $(\mathbb{N} \cup\{-1\})^{n}$ est tel que $x^{I}$ est une fonction de Casimir pour la partie quadratique $\Pi^{0}$, et le champ de bivecteurs $\Pi^{I}$ est quadratique diagonal.

Remarque. En général, une même classe de conjuguaison comporte plusieurs structures de Poisson distinctes sous forme normale au sens de Dufour-Wade. Si $\Pi$ est sous forme normale, alors on a $\left[\Pi^{0}, \Pi\right]=0$.

La preuve du théorème de Dufour et Wade repose sur le lemme suivant.

LEMME 2.2. Soit $x^{I} B$ un champ de bivecteurs holomorphe non-résonnant, avec $I \in$ $(\mathbb{N} \cup\{-1\})^{n}$, et $B$ champ de bivecteurs quadratique diagonal. Supposons $\left[\Pi^{0}, x^{I} B\right]=0$ ainsi que I contient au plus une composante négative. Alors il existe un champ de vecteurs linéaire diagonal $Z$, tel que $x^{I} Z$ est holomorphe, et tel que

$$
\left[\Pi^{0}, x^{I} Z\right]=x^{I} B .
$$

Ce lemme est la traduction de Dufour-Wade [6, proposition 3], dans les notations adoptées ici. Dans les notations adoptées ici l'hypothèse $(\mathrm{H})$ se formule de la manière suivante.

Définition 2.5. (Hypothèse $(\mathrm{H})$ sur la partie quadratique $\Pi^{0}$ ) Pour tout

$$
I=\left(I_{1}, \ldots, I_{i-1},-1, I_{i+1}, \ldots, I_{j-1},-1, I_{j+1}, \ldots, I_{n}\right)
$$

avec $I_{l} \in \mathbb{N}$ pour tout $l \neq i, j$, tel que

$$
\left.\frac{1}{x^{I}} X_{\Pi^{0}}\left(x^{I}\right)\right) \wedge Y_{i} \wedge Y_{j}=0
$$

le monôme $x^{I}$ est une fonction de Casimir pour la partie quadratique $\Pi^{0}$.

L'hypothèse $(\mathrm{H})$ signifie que tout champ de bivecteurs $x^{I} B$, avec $I \in(\mathbb{N} \cup\{-1\})^{n}$ admettant deux composantes négatives égales à -1 , satisfaisant la relation $\left[\Pi^{0}, x^{I} B\right]=0$, est résonnant.

\section{Le résultat principal}

3.1. Algèbres de Lie associées et petits diviseurs. Soit $\Pi$ une structure de Poisson holomorphe de 1 -jet nul en $0 \in \mathbb{C}^{n}$. La présente section est consacrée à l'introduction de plusieurs objets associés à sa partie quadratique $\Pi^{0}:=(1 / 2) \sum_{i \neq j} a_{i j} Y_{i} \wedge Y_{j}, a_{i j}=$ $-a_{j i}$, supposée diagonale.

(i) Associons à $\Pi^{0}$ les champs de vecteurs linéaires diagonaux

$$
S_{i}:=\sum_{j=1}^{n} a_{i j} Y_{j},
$$


ainsi que l'algèbre de Lie abélienne $\mathbb{S}$ engendrée par les champs $S_{i}$ (pour le crochet de Lie).

(ii) Notons $\mathfrak{S}$ l'espace vectoriel des champs de bivecteurs quadratiques sur $\mathbb{C}^{n}$ s'écrivant

$$
\sum_{i=1}^{n} B_{i} \wedge Y_{i} \quad \text { avec } \quad B_{i}:=\sum_{j=1}^{n} b_{i j} Y_{j} \in \mathbb{S}, \quad b_{i j}=-b_{j i} .
$$

(iii) Introduisons l'anneau des intégrales premières formelles $\widehat{\mathcal{O}}^{S}$ (respectivement holomorphes $\mathcal{O}^{S}$ ) de $\mathbb{S}$.

Remarque. Une fonction holomorphe $f$ appartient à $\widehat{\mathcal{O}}^{S}$, si pour tout $1 \leq i \leq n$ on a $\mathcal{L}_{S_{i}}(f)=0$. L'ensemble des fonctions de Casimir formelles de $\Pi^{0}$ est égal à $\widehat{\mathcal{O}}^{S}$.

(iv) Un terme de la forme $x^{J} Y_{j}$ (avec éventuellement $J_{j}=-1$ ) est appellé résonnant, s'il commute avec les champs $S_{i}$, c'est-à-dire si $\left[S_{i}, x^{J} Y_{j}\right]=0$ pour tout $1 \leq i \leq n$.

(v) On note $\mathcal{P}^{m, 2 m}$ l'espace vectoriel des champs de vecteurs polynomiaux s'écrivant $\sum_{m+1 \leq|I| \leq 2 m} x^{I} L^{I}$, avec $L$ champ de vecteurs linéaire diagonal, $I$ multiindice (avec éventuellement une composante égale à -1 pourvu que $x^{I} L^{I}$ reste holomorphe). De même, notons $\widetilde{\mathcal{P}}^{m, 2 m}$ l'espace vectoriel des champs de bivecteurs holomorphes s'écrivant $\sum_{m+1 \leq|I| \leq 2 m} x^{I} B^{I}$, avec $B^{I}$ champ de bivecteurs quadratique diagonal, $I$ un multi-indice (avec éventuellement une ou deux composantes égales à -1 pourvu que le terme $x^{I} B^{I}$ reste holomorphe).

Remarque. Les éléments de $\mathcal{P}^{m, 2 m}$ sont d'ordre $\geq m+2$ et de degré $\leq 2 m+1$. Les éléments de $\widetilde{\mathcal{P}}^{m, 2 m}$ sont d'ordre $\geq m+3$ et de degré $\leq 2 m+2$.

(vi) Les applications $S_{i} \longmapsto\left[S_{i},.\right]$ définissent des représentations semi-simples $\rho^{m}$ (respectivement $\tilde{\rho}^{m}$ ) de l'algèbre de Lie $\mathbb{S}$ dans $\mathcal{P}^{m, 2 m}$ (respectivement $\widetilde{\mathcal{P}}^{m, 2 m}$ ).

(vii) Une forme linéaire $\alpha$ sur $\mathbb{S}$ est un poids pour la représentation $\rho^{m}$ s'il existe $X \in \mathcal{P}^{m, 2 m} \neq 0$ tel que $\alpha\left(S_{i}\right) X=\left[S_{i}, X\right], 1 \leq i \leq l$. L'ensemble de ces champs $X$ forme l'espace de poids associé $\mathcal{P}_{\alpha}^{m, 2 m}$.

Définition 3.1. Soit $\alpha$ un poids non nul pour la représentation $\rho^{m}$. Posons $|\alpha|:=$ $\max _{1 \leq j \leq n}\left|\alpha\left(S_{j}\right)\right|$. Pour $k \geq 1$, définissons le nombre de Bruno $\omega_{k}$ associé à $\Pi^{0}$ :

$$
\omega_{k}:=\inf \left\{|\alpha|: \alpha \text { poids non nul pour } \rho^{\left(2^{j}\right)}, 2 \leq j \leq k\right\} .
$$

Définition 3.2. (Condition diophantienne (D)) On dit que $\Pi^{0}$ vérifie la condition (D) si on a

$$
\sum_{k \geq 0} \frac{-\ln \left(\omega_{k}\right)}{2^{k}}<+\infty
$$

Remarque. La condition diophantienne (D) ne dépend pas du choix de la norme dont on muni l'espace des formes linéaires sur $\mathbb{S}$ pour définir les nombres de Bruno.

3.2. La condition algébrique sur la forme normale. Soit $\Pi$ une structure de Poisson à 1-jet nul. Supposons que sa partie quadratique $\Pi^{0}$ satisfait l'hypothèse $(\mathrm{H})$, garantissant l'existence d'une forme normale formelle. Soient $\mathbb{S}, \mathfrak{S}$ et $\widehat{\mathcal{O}}^{S}$ telles que définies en section 3.1 . 
Définition 3.3. (Condition (C)) On dit qu'une forme normale formelle (au sens de DufourWade) de $\Pi$ satisfait la condition $(\mathrm{C})$ si elle appartient à $\widehat{\mathcal{O}}^{S} \otimes \mathfrak{S}$, c'est-à-dire s'écrit $\sum_{I} x^{I} \Pi^{I}$, où $x^{I} \in \widehat{\mathcal{O}}^{S}$ et $\Pi^{I} \in \mathfrak{S}$.

Remarque. Si $x^{I} \Pi^{I}$, avec $\Pi^{I}$ quadratique diagonal, est un terme d'une forme normale de Dufour-Wade générale, alors il se peut que deux coordonnées de $I$ soient égales à -1 . Montrons que ceci n'est pas le cas sous la condition (C) : si $x^{I} \Pi^{I} \in \widehat{\mathcal{O}}^{S} \otimes \mathfrak{S}$, alors $I$ appartient à $\mathbb{N}^{n}$. En effet, soit $x^{I} \Pi^{I} \in \widehat{\mathcal{O}} \otimes \mathfrak{S}$, et posons

$$
x^{I} \Pi^{I}:=x^{I} \frac{1}{2} \sum_{i \neq j} b_{i j} Y_{i} \wedge Y_{j}
$$

avec $b_{i j}=-b_{j i}$. Raisonnons par l'absurde, et supposons que la $i_{0}$-ème coordonnée de $I$ soit égale à -1 . Puisque les monômes $x^{I} a_{i j} Y_{i} \wedge Y_{j}$ et $-x^{I} a_{j i} Y_{j} \wedge Y_{i}$ doivent être holomorphes, tous les coefficients de la matrice $\left(a_{i j}\right)$ sont nuls s'ils n'appartiennent pas à la $i_{0}$-ème ligne ou colonne. Comme la matrice $\left(a_{i j}\right)$ est antisymétrique et non nulle, il existe un indice $j_{0} \neq i_{0}$ avec $a_{i_{0} j_{0}} \neq 0$. Dans la $j_{0}$-ème ligne de la matrice $\left(a_{i j}\right)$, seul $a_{j_{0} i_{0}}$ est alors non nul. De plus, le monôme $x^{I}$ est alors intégrale première du champ de vecteurs $a_{j_{0} i_{0}} Y_{i_{0}}$, mais ceci est absurde, puisque la $i_{0}$-ème composante de $I$ est égale à -1 .

Remarque. $\mathrm{Si} \operatorname{dim} \mathbb{S}=n$, alors la forme normale de Dufour-Wade se réduit à la partie quadratique. En effet, l'anneau $\widehat{\mathcal{O}}^{S}$ des intégrales premières formelles de $\mathbb{S}$ est alors réduit aux constantes.

Proposition 3.1. Si une forme normale formelle de $\Pi$ satisfait à la condition $(C)$, alors toutes les autres formes normales formelles de $\Pi$ satisfont également à $(C)$.

Pour la preuve de la proposition 3.1, on a besoin des deux lemmes suivants.

Lemme 3.1. Soit $Z$ un champ de vecteurs, $\Pi^{\prime} \in \mathfrak{S}$ et $I \in \mathbb{Z}^{n}$ un multi-indice. Posons $\Pi^{\prime}:=\sum_{i=1}^{n} A_{i}^{\prime} \wedge Y_{i}$ et $A_{i}^{\prime}:=\sum_{k=1}^{n} a_{i k}^{\prime} Y_{k}$. Alors,

$$
\left[Z, x^{I} \Pi^{\prime}\right]=2 x^{I} \sum_{i=1}^{n}\left[Z, A_{i}^{\prime}\right] \wedge Y_{i}+\mathcal{L}_{Z}\left(x^{I}\right) \sum_{i=1}^{n} A_{i}^{\prime} \wedge Y_{i} .
$$

Preuve du lemme 3.1. On a, d'après (2.2),

$$
\left[Z, x^{I} \Pi^{\prime}\right]=\sum_{i=1}^{n}\left[Z, x^{I} A_{i}^{\prime}\right] \wedge Y_{i}-x^{I} \sum_{i=1}^{n}\left[Z, Y_{i}\right] \wedge A_{i}^{\prime} .
$$

En remplaçant pour tout $1 \leq i \leq n, A_{i}^{\prime}$ par $\sum_{k=1}^{n} a_{i k}^{\prime} Y_{k}$ et en faisant entrer les coefficients $a_{i k}^{\prime}$ dans le crochet, on obtient

$$
x^{I} \sum_{i=1}^{n}\left[Z, Y_{i}\right] \wedge A_{i}^{\prime}=x^{I} \sum_{1 \leq k, i \leq n}\left[Z, a_{i k}^{\prime} Y_{i}\right] \wedge Y_{k} .
$$

Par antisymétrie de la matrice $\left(a_{i k}^{\prime}\right)$, ceci devient $-x^{I} \sum_{k=1}^{n}\left[Z, A_{k}^{\prime}\right] \wedge Y_{k}$. Au total on obtient

$$
\left[Z, x^{I} \Pi^{\prime}\right]=2 x^{I} \sum_{i=1}^{n}\left[Z, A_{i}^{\prime}\right] \wedge Y_{i}+\sum_{i=1}^{n} \mathcal{L}_{Z} x^{I} A_{i}^{\prime} \wedge Y_{i}
$$


LEMME 3.2. Soit $x^{J} Y_{j}$ holomorphe et résonnant tel qu'en section 3.1 (iv), et soit $x^{I} \Pi^{I} \in$ $\widehat{\mathcal{O}}^{S} \otimes \mathfrak{S}$, c'est-à-dire $x^{I} \in \widehat{\mathcal{O}}^{S}$ et $\Pi^{I} \in \mathfrak{S}$. Alors $\left[x^{J} Y_{j}, x^{I} \Pi^{I}\right] \in \widehat{\mathcal{O}}^{S} \otimes \mathfrak{S}$.

Preuve du lemme 3.2. Rappelons que $\Pi^{I} \in \mathfrak{S}$ signifie que $\Pi^{I}=\sum_{i=1}^{n} A_{i}^{I} \wedge Y_{i}$ avec $A_{i}^{I}:=\sum_{j=1}^{n} a_{i j}^{I} Y_{j}$, avec $a_{i j}^{I}=-a_{j i}^{I}$ et $A_{i}^{I} \in \mathbb{S}$ pour tout $1 \leq i \leq n$. D'après le lemme 3.1,

$$
\left[x^{J} Y_{j}, x^{I} \Pi^{I}\right]=2 x^{I} \sum_{i=1}^{n}\left(\left[x^{J} Y_{j}, A_{i}^{I}\right] \wedge Y_{i}\right)+\left(\mathcal{L}_{x^{J} Y_{j}} x^{I}\right) \Pi^{I} .
$$

La première expression du second membre est nulle, car $A_{i}^{I} \in \mathbb{S}$ et $x^{J} Y_{j}$ est résonnant, tandis que la deuxième expression du second membre appartient à $\widehat{\mathcal{O}}^{S} \otimes \mathfrak{S}$ : en effet, $\mathcal{L}_{\left(x^{J} Y_{j}\right)} x^{I}=I_{j} x^{I+J}$, où $I_{j}$ désigne la $j$-ème coordonnée de $I$. Il suit $\mathcal{L}_{S_{i}}\left(x^{I+J}\right)=0$ pour tout $1 \leq i \leq n$. Reste à montrer : si $I_{j} \neq 0$, alors le multi-indice $I+J$ n'a pas de composante strictement négative. Ceci suit du fait que $I+J$ a une composante strictement négative (la $j$-ème) si et seulement si la $j$-ème coordonnée de $J$ est égale à -1 ainsi que $I_{j}=0$.

Preuve de la proposition 3.1. Supposons $\Pi \in \widehat{\mathcal{O}}^{S} \otimes \mathfrak{S}$, c'est-à-dire

$$
\Pi=\sum_{J} x^{J} \Pi^{J}
$$

avec $x^{J} \in \widehat{\mathcal{O}}^{S}$ et $\Pi^{J} \in \mathfrak{S}$ et soit $\Psi^{*}(\Pi)$ une autre forme normale formelle (au sens de Dufour-Wade) de $\Pi$. Montrons que $\Psi^{*}(\Pi) \in \widehat{\mathcal{O}}^{S} \otimes \mathfrak{S}$.

Soit $\left(\Psi_{n}\right)_{n \geq 1}, \Psi_{n} \equiv \exp X_{n}$, la suite de transformations formelles définie récursivement par

$$
\Psi_{1}:=\Psi, \quad \Psi_{n+1}:=\exp \left(-Z_{n+1}\right) \circ \Psi_{n} \quad \text { pour } n>1,
$$

où $Z_{n+1}$ est la somme des termes de degré $n+1$ du champ de vecteurs (formel) $X_{n}$ qui sont résonnants (dans le sens de la section 3.1 (iv)).

Il suit alors de (2.5) que par construction, le champ de vecteurs $X_{n}$ n'a pas de terme résonnant de degré inférieur où égal à $n$. A la limite, on obtient une transformation formelle $\Psi_{\infty}=\exp \left(X_{\infty}\right)$, où le champ de vecteurs formel $X_{\infty}$ est composé exclusivement de termes non-résonnants, telle que

$$
\Psi=\left(\exp \left(-Z_{2}\right)^{-1} \circ \exp \left(-Z_{3}\right)^{-1} \circ \cdots\right) \circ \Psi_{\infty}
$$

avec $Z_{n}$ résonnant homogène de degré $n$. Par le Lemme 3.3, prouvé un peu plus loin, on a $\Psi_{\infty}^{*}(\Pi)=\Pi$. Il suit

$$
\Psi^{*}(\Pi)=\left(\exp \left(-Z_{2}\right)^{-1} \circ \exp \left(-Z_{3}\right)^{-1} \circ \cdots\right)^{*}(\Pi) .
$$

Or, de (2.5) et du lemme 3.2 on déduit que pour chaque transformation $\exp \left(Z_{n}\right)^{-1}$ on a

$$
B \in \widehat{\mathcal{O}}^{S} \otimes \mathfrak{S} \Rightarrow\left(\exp \left(-Z_{n}\right)^{-1}\right)^{*}(B)=\exp \left(Z_{n}\right)^{*}(B) \in \widehat{\mathcal{O}}^{S} \otimes \mathfrak{S} .
$$

Comme par hypothèse, $\Pi \in \widehat{\mathcal{O}}^{S} \otimes \mathfrak{S}$, il suit avec (3.2) qu'on a bien $\Psi^{*}(\Pi) \in \widehat{\mathcal{O}}^{S} \otimes \mathfrak{S}$. 
LEMME 3.3. $\Psi_{\infty}^{*}(\Pi)=\Pi$.

Preuve. Etablissons d'abord que $\Psi_{\infty}^{*}(\Pi)$ est une forme normale au sens de DufourWade. Pour cela, constatons que pour tout $n \geq 1$, la structure de Poisson $\Psi_{n}^{*}(\Pi)$ est sous forme normale : en effet, $\Psi_{1}^{*}(\Pi)\left(=\Psi^{*}(\Pi)\right)$ est une forme normale, et, puisque $Z_{n}$ est résonnant, (2.5) et le lemme 2.1 impliquent que l'image d'une forme normale par $\exp \left(-Z_{n}\right)$ est une forme normale.

Passons maintenant à la démonstration de $\Psi_{\infty}^{*}(\Pi)=\Pi$. Rappelons $\Psi_{\infty}=\exp \left(X_{\infty}\right)$ et posons

$$
X_{\infty}:=\sum_{|I|>0} x^{I} X_{I}
$$

avec $X_{I}$ champ de vecteurs linéaire diagonal, et $I$ multi-indice (avec éventuellement une composante égale à -1 ). Rappelons que chaque terme apparaissant effectivement dans la somme est non-résonnant. Soit $x^{K} \Pi_{K}$ un monôme de plus petit degré du champ de bivecteurs $\Psi_{\infty}^{*}(\Pi)-\Pi$. Montrons qu'il est nul. Par $\Psi_{\infty}=\exp \left(X_{\infty}\right)$ et $(2.5), x^{K} \Pi_{K}$ est une somme de termes de la forme

$$
\left[x^{I} X^{I}, x^{J} \Pi^{J}\right]
$$

avec $I+J=K$, où $x^{J} \Pi^{J}$ est un terme provenant de $\Pi$, c'est-à-dire du deuxième membre de (3.1), et $x^{I} X^{I}$ est un terme (non-résonnant) du second membre de (3.3). D'après le lemme 2.1, le terme (3.4) est non-résonnant. On en déduit que si $x^{K} \Pi_{K}$ est non nul, alors $x^{K} \Pi_{K}$ est non-résonnant. Mais ceci contredit le fait, établi plus haut, que $\Psi_{\infty}^{*}(\Pi)$ est une forme normale au sens de Dufour-Wade de $\Pi$. Donc $x^{K} \Pi_{K}$ est nul.

Remarque. Le fait que l'hypothèse sur la forme normale soit intrinsèque permettra, dans la démonstration du théorème de normalisation qui suit, de normaliser vers une forme normale qui peut différer de la forme normale initialement donnée.

\subsection{Un théorème de normalisation et son cas particulier quadratique.}

THÉORÈme 3.1. (De normalisation) Soit $\Pi$ une structure de Poisson à 1-jet nul en $0 \in \mathbb{C}^{n}$. On suppose que sa partie quadratique $\Pi^{0}$ est diagonale et satisfait l'hypothèse $(H)$. On suppose également que les formes normales formelles de $\Pi$ satisfont l'hypothèse $(C)$, et que $\Pi^{0}$ satisfait l'hypothèse $(D)$. Alors $\Pi$ est holomorphiquement conjuguée à une forme normale (i.e. il existe un biholomorphisme $\Phi:\left(\mathbb{C}^{n}, 0\right) \rightarrow\left(\mathbb{C}^{n}, 0\right)$ tel que $\left.\Phi^{*} \Pi \in \mathcal{O}^{S} \otimes \mathfrak{S}\right)$.

Corollaire 3.1. (De quadratisation) Soit $\Pi$ une structure de Poisson formellement quadratisable. Supposons que la partie quadratique $\Pi^{0}$ de $\Pi$ est diagonale et satisfait l'hypothèse $(H)$, ainsi que la condition diophantienne $(D)$. Alors $\Pi$ est holomorphiquement quadratisable.

En effet, on peut montrer que si une forme normale de $\Pi$ est quadratique, alors toutes les formes normales de $\Pi$ sont quadratiques. Par le théorème 3.1, on peut alors conjuguer $\Pi$ à $\Pi^{0}$ par un biholomorphisme.

Rapport avec le résultat de Dufour-Zung [7, théorème 5.7.5] On peut déduire de la démonstration du résultat cité, qu'une structure de Poisson $\Pi$ à 1-jet nul est formellement 
quadratisable si la partie linéaire diagonale $S$ du rotationnel de $\Pi$ n'admet de résonances autres que celles qui sont engendrées par le monôme $x_{1} \cdots x_{n}$.

D'après le résultat cité, une structure de Poisson $\Pi$ à 1-jet nul est holomorphiquement quadratisable si $S$ satisfait en plus la condition $(\omega)$ de Bruno, c'est-à-dire $-\sum_{k>0}\left(\ln \omega_{k}^{\prime} / 2^{k}\right)<\infty$, où on a posé $\omega_{k}^{\prime}:=\min \left\{|S . I|: S . I \neq 0,1 \leq|I| \leq 2^{k}\right\}$, avec $S . I:=\left(1 / x^{I}\right) \mathcal{L}_{S}\left(x^{I}\right)$. Soient $S_{1}, \ldots, S_{n}$ les champs de vecteurs linéaires diagonaux associés à partie quadratique $\Pi^{0}$ de $\Pi$ (cf. section 3.1). On a $S=S_{1}+\cdots+S_{n}$. Le champ $S$ appartient donc à l'algèbre $\mathbb{S}$. Puisque la la conditon diophantienne (D) du corollaire 3.1 porte sur l'algèbre $\mathbb{S}$ tout entière, elle est plus faible que la condition diophantienne $(\omega)$ de Bruno sur le seul champ $S$ (cf. Stolovitch [8]). Donnons un exemple en dimension $2:$ soit $\lambda:=\sum_{n>0}\left(c_{n} / \mu^{n !}\right)$ où $\mu$ est un entier $>1$ et $c_{n}$ un entier compris entre 0 et 9 non constament nul à partir d'un certain indice $n$. Soit $\Pi^{0}$ la structure de Poisson quadratique de matrice

$$
\left(\begin{array}{rrr}
0 & 1 & 0 \\
-1 & 0 & -\lambda \\
0 & \lambda & 0
\end{array}\right)
$$

Le rotationnel $S=-Y_{1}+(1+\lambda) Y_{2}-\lambda Y_{3}$ ne vérifie pas la condition diophantienne de Bruno. Mais la condition diophantienne (C) du corollaire 3.1 est satisfaite.

3.4. Interprétation géométrique. Soit $\Pi$ une structure de Poisson à 1 -jet nul en $0 \in \mathbb{C}^{n}$ dont la partie quadratique $\Pi^{0}$ est diagonale.

Stolovitch, dans [8, les sections $2.2,5.3,5.4]$ a établi la proposition suivante.

PROPOSITION 3.2.

(i) Si l'anneau $\widehat{\mathcal{O}}^{S}$ n'est pas réduit à $\mathbb{C}$, alors $\widehat{\mathcal{O}}^{S}$ est une $\mathbb{C}$-algèbre de type fini, et il existe des monômes $u_{1}, \ldots, u_{p}$ tel que $\widehat{\mathcal{O}}^{S}=\mathbb{C}\left[\left[u_{1}, \ldots, u_{p}\right]\right]$.

(ii) Les relations entre les $u_{i}$ définissent une variété algébrique $\mathcal{C}_{S}$ dans $\mathbb{C}^{p}$, de dimension $s$, où $s$ est le nombre maximal de $u_{i}$ algébriquement indépendants. L'application $\pi: \mathbb{C}^{n} \rightarrow \mathbb{C}^{p}$ définie par $\pi(x)=\left(u_{1}(x), \ldots, u_{p}(x)\right)$ est une fibration singulière au-dessus de $\mathcal{C}_{S}$. De plus chaque $S_{i}$ est tangent aux fibres de $\pi$.

COROllaire 3.2. Sous les hypothèses du théorème 3.1, il existe au voisinage de 0 un système de coordonnées holomorphes tel que $\Pi$ appartient à $\mathcal{O}^{S} \otimes \mathfrak{S}$. Soit, dans ces coordonnées, $\Pi_{\mid \mathcal{F}}$ la restriction de $\Pi$ à une fibre $\mathcal{F}$ de $\pi$. On a alors

$$
\Pi_{\mid \mathcal{F}}=\Pi_{\mid \mathcal{F}}^{\mathcal{F}}
$$

où $\Pi^{\mathcal{F}}$ est une structure de Poisson quadratique diagonale appartenant à $\mathfrak{S}$ (cf. section 3.1) qui dépend uniquement de la fibre $\mathcal{F}$. De plus, sur chaque fibre $\mathcal{F}$, pour toute fonction holomorphe $f$, le champ hamiltonien de $f$ relativement à $\Pi$ est tangent à $\mathcal{F}$.

On en déduit que les feuillets symplectiques de $\Pi$ (cf. introduction) sont, au voisinage de 0 , inclus dans les fibres de $\pi$. Ces feuillets symplectiques ont la dimension de l'algèbre $\mathbb{S}$. Si la dimension de $\mathbb{S}$ est égale à $n-s$, alors aux points réguliers de $\pi$, les 
feuillets symplectiques de $\Pi$ coïncident avec les fibres. L'hypothèse $(\mathrm{C})$ sur la forme normale formelle implique que l'ensemble des fonctions de Casimir formelles de la forme normale coïncide avec celui de la partie quadratique. Dans certains cas, ceci admet une réciproque : Soit $\Pi$ un structure de Poisson à 1 -jet nul en $0 \in \mathbb{C}^{n}$, dont la partie quadratique est diagonale et satisfait l'hypothèse $(\mathrm{H})$. Rappelons que l'on note $s$ le nombre maximal de générateurs de $\widehat{\mathcal{O}}^{S}$ qui sont algébriquement indépendants. La structure de Poisson $\Pi$ est holomorphiquement normalisable sous les hypothèses suivantes.

Corollaire 3.3. Supposons l'algèbre $\mathbb{S}$ de dimension $n-s$, et supposons qu'il existe un système de coordonnées formelles dans lequel l'ensemble des fonctions de Casimir de $\Pi$ est égal à $\widehat{\mathcal{O}}^{S}$ (l'ensemble des fonctions de Casimir de $\Pi^{0}$ ). Supposons en outre que la partie quadratique $\Pi^{0}$ vérifie la condition diophantienne $(D)$. Alors $\Pi$ est holomorphiquement normalisable.

Esquissons rapidement, à travers les deux lemmes suivants, comment on peut se ramener au théorème 3.1 , c'est-à-dire pourquoi il existe un changement de variable formel $\widehat{\Phi}$ tel que l'on ait $\widehat{\Phi}^{*}(\Pi) \in \widehat{\mathcal{O}}^{S} \otimes \mathfrak{S}$.

LEMME 3.4. Dans un système de coordonnées formelles dans lequel l'ensemble des fonctions de Casimir de $\Pi$ est égal à $\widehat{\mathcal{O}}^{S}$, $\Pi$ s'écrit sous la forme $\sum_{|I|>0} x^{I} \Pi^{I}$ où $\Pi^{I} \in \mathfrak{S}$.

Esquisse de preuve. Situons nous dans un système de coordonnées formelles dans lequel l'ensemble des fonctions de Casimir de $\Pi$ est égal à $\widehat{\mathcal{O}}^{S}$. Choisissons une famille $\left(u_{1}, \ldots, u_{p}\right)$ de monômes générateurs de $\mathbb{S}$ et notons $\mathcal{F}_{y}$ les fibres (de dimension $n-s$ ) de l'application $\pi: \mathbb{C}^{n} \rightarrow \mathbb{C}^{p}, x \rightarrow\left(u_{1}(x), \ldots, u_{p}(x)\right)=y(x)$. Posons

$$
\Pi:=\Pi^{0}+\sum_{|I|>0} x^{I} \Pi^{I}, \quad \Pi^{I}:=\sum_{i} B_{i}^{I} \wedge Y_{i} \quad \text { et } \quad B_{i}^{I}=\sum_{j} b_{i j}^{I} Y_{j}, \quad b_{i j}^{I}=-b_{j i}^{I} .
$$

On a alors

$$
X_{\Pi}\left(x_{i}\right)=x_{i} \sum_{|I|>0} x^{I} B_{i}^{I}, \quad 1 \leq i \leq n .
$$

Or, comme les $u_{j}$ sont des fonctions de Casimir, on doit avoir $\mathcal{L}_{X_{\Pi}\left(x_{i}\right)}\left(u_{j}\right)=0$ pour tout $1 \leq j \leq p$ et $1 \leq i \leq n$. Ceci signifie que $B_{i}^{I}$ est tangent à $\mathcal{F}_{y}$ pour tout $I$ et tout $y$. L'appartenance de $B_{i}^{I}$ à $\mathbb{S}$ suit alors du fait que la dimension de $\mathcal{F}_{y}$ soit $n-s$ si $y$ est une valeur réguière de $\pi$. D'où $\Pi^{I} \in \mathfrak{S}$.

Corollaire 3.4. Si $\Pi$ s'écrit $\sum_{|I|>0} x^{I} \Pi^{I}$, où $\Pi^{I} \in \mathfrak{S}$, il existe une transformation normalisante formelle $\widehat{\Phi}$ qui préserve les fonctions de Casimir.

Esquisse de preuve. Soit $x^{I_{0}} \Pi^{I_{0}}$ un terme non-résonnant de plus petit degré. De l'identité de Jacobi $[\Pi, \Pi]=0$ et du lemme 2.2 suit l'existence d'un champ de vecteurs linéaire diagonal $Z_{D}$ tel que $\left[\Pi^{0}, X^{I_{0}} Z_{D}\right]=x^{I_{0}} \Pi^{I_{0}}$. Pour montrer l'existence d'une transformation normalisante préservant les fonctions de Casimir il suffit de montrer que $Z_{D}$ appartient à l'algèbre $\mathbb{S}$ : en effet, il suit de $Z_{D} \in \mathbb{S}$ que $\exp (Z)$ préserve le fonctions de Casimir, et d'après le lemme 3.4, $(\exp (Z))^{*}(\Pi)$ s'écrit encore sous forme $\sum_{I^{\prime}} x^{I^{\prime}} \Pi^{I^{\prime}}$, 
où $\Pi^{I^{\prime}} \in \mathfrak{S}$. Montrons que $Z_{D}$ appartient à $\mathbb{S}$. Par (2.9),

$$
\Pi^{I_{0}}=Z_{D} \wedge \frac{1}{x^{I_{0}}} X_{\Pi^{0}}\left(x^{I_{0}}\right)
$$

où $X_{\Pi^{0}}\left(x^{I_{0}}\right)$ désigne le champ hamiltonien de $x^{I_{0}}$ relativement à $\Pi^{0}$. Or par hypothèse, $\Pi^{I_{0}} \in \mathfrak{S}$, donc tout champ hamiltonien relativement à $\Pi^{I_{0}}$ est parallèle à un élément de $\mathbb{S}$. Comme $X_{\Pi^{0}}\left(x^{I_{0}}\right) \in \mathbb{S}$, il suit avec (3.5) que $Z_{D} \in \mathbb{S}$.

Remarque. On définit une enveloppe diophantienne $\widetilde{\mathbb{S}}$ de $\mathbb{S}$ comme une algèbre de Lie de champs de vecteurs linéaires diagonaux contenant $\mathbb{S}$ telle que :

(i) l'ensemble des intégrales premières formelles communes des éléments de $\widetilde{\mathbb{S}}$ coïncide avec avec $\widehat{\mathcal{O}}^{S}$;

(ii) il existe une constante $c$ tel que pour tout champ de vecteurs formel $P$ d'ordre 2 on ait $\max _{S \in \widetilde{\mathbb{S}}}(|\alpha(S)|) \leq \max _{S \in \mathbb{S}}(|\alpha(S)|)$, où $\alpha(S)$ est un nombre réel tel que $\alpha(S) P=[S, P]$ (voir Stolovitch $[8])$.

Le théorème 3.1 reste vrai si on remplace l'algèbre $\mathbb{S}$ par une enveloppe diophantienne $\widetilde{\mathbb{S}}$ de celle ci.

\subsection{Esquisse de la démonstration du théorème de normalisation.}

L'équation cohomologique et la majoration de ses solutions. La démonstration repose sur une méthode de Newton inventée par Kolmogorov et Arnol'd : supposons П normalisé au degré $m$. On note alors

$$
\Pi=\Pi^{m}+B_{*}^{m}+B_{0}^{m}+R^{m},
$$

où $\Pi^{m}$ désigne la partie de $\Pi$ qui est déja sous forme normale, c'est-à-dire la somme des termes de degré inférieur à $m+3$ de $\Pi$. On désigne par $B_{*}^{m}$ (respectivement $B_{0}^{m}$ ) la somme des termes non-résonnants (respectivement résonnants) de degré compris entre $m+3$ et $2 m+2$. Par $R^{m}$ on entend la somme des termes de degré supérieur à $2 m+2$.

La transformation $\exp \left(Z^{m}\right)$, avec $Z^{m}$ champ de vecteurs polynomial d'ordre $m+1$ et de degré $2 m+1$, normalise $\Pi$ au degré $2 m+2$ si et seulement si $Z^{m}$ est solution de l'équation cohomologique

$$
J^{2 m+2}\left(\left[\Pi^{m}, Z^{m}\right]\right)=B_{*}^{m} .
$$

Il reste à choisir $Z^{m}$ de manière que la limite (formelle) de la suite de transformations $\exp \left(Z^{2^{k}}\right) \circ \exp \left(Z^{2^{k-1}}\right) \circ \cdots \circ \exp Z^{1}$ soit convergente. Le point clef est l'assertion suivante.

LEMME 3.5. Il existe des constantes positives $\eta$ et e, déterminées par $\Pi^{0}$, telles que, pour :

(i) tout entier $m>0$;

(ii) toute forme normale de Dufour-Wade $\Pi^{m}$, de partie quadratique $\Pi^{0}$, polynomiale de degré inférieur à $m+3$, satisfaisant à la condition $(C)$; et

(iii) toute $B_{*}^{m} \in \widetilde{\mathcal{P}}^{m, 2 m}$ somme de monômes non-résonnants telle que la relation de compatibilité $J^{2 m+4}\left(\left[\Pi^{m}+B_{*}^{m}, \Pi^{m}+B_{*}^{m}\right]\right)=0$ soit satisfaite ; 
il existe une solution $Z^{m} \in \mathcal{P}^{m, 2 m}$ de l'équation cohomologique (3.6) telle que, pour tout $r \in] 1 / 2,1]$,

$$
\left\|\Pi^{m}-\Pi^{0}\right\|_{r}<\eta \text { et }\left\|D\left(\Pi^{m}-\Pi^{0}\right)\right\|_{r}<\eta \Longrightarrow\left\|Z^{m}\right\|_{r} \leq \frac{m e}{\omega_{m}^{3}}\left\|B_{*}^{m}\right\|_{r},
$$

où on a posé $\omega_{m}=\inf \left\{|\alpha|: \alpha\right.$ poids non nul pour la représentation $\left.\rho^{k}, 1 \leq k \leq m\right\}$.

Pour démontrer le théorème précédent on décompose d'abord l'équation cohomologique (3.6) selon les poids non nuls $\beta$ des représentations semi-simples $\rho^{m}$ et $\tilde{\rho}^{m}$. Il s'agit alors de majorer les solutions $Z_{\beta}^{m}$ des équations

$$
J^{2 m+2}\left(\left[\Pi^{m}, Z_{\beta}^{m}\right]\right)=B_{* \beta}^{m},
$$

où $B_{* \beta}^{m}$ est la projection sur l'espace de poids $\widetilde{\mathcal{P}}_{\beta}^{m, 2 m}$.

Deux cas distincts. Le lemme 2.2 regroupe deux situations entièrement distinctes : si $I$ n'admet pas de composantes strictement négatives, alors la solution $x^{I} Z$ de (2.13) est définie modulo l'addition d'un multiple de $X_{\Pi^{0}}\left(x^{I}\right)$ (le champ hamiltonien de $x^{I}$ relativement à $\Pi^{0}$ ).

Si $I$ admet une composante égale à -1 , par exemple la $i_{0}$-ème, alors $\Pi^{I}$ est le produit exterieur de $Y_{i_{0}}$ avec un champ de vecteurs linéaire diagonal. Le champ de vecteurs analytique $x^{I} Z$ qui satisfait (2.13) est unique de la forme $c x^{I} Y_{i_{0}}, c \in \mathbb{C}$.

Démarche adopté pour majorer les solutions de (3.7). On établit d'abord (en section 4.2) l'existence d'une transformation $\exp \left(Z_{\beta-}^{m}\right)$ permettant de ramener le problème de la majoration des solution de (3.7) au cas où $B_{* \beta}^{m}$ est une somme de monômes $x^{I} \Pi^{I}$, avec $\Pi^{I}$ quadratique diagonal, et $I \in \mathbb{N}^{n}$ (ne comportant pas de composantes strictement négatives).

Deuxième partie (section 4.3) : soit $1 \leq i_{m} \leq n$ un indice tel que $\max _{1 \leq i \leq n}\left|\beta_{i}\right|:=|\beta|=$ $\left|\beta_{i_{m}}\right|$. Notons $K_{i_{m}}:=(0, \ldots, 0,1,0, \ldots 0)$ le multi-indice dont toutes les composantes sont nulles, à l'exception de la $i_{m}$-ème, qui est égale à 1 . L'holomorphie du champ de vecteurs $\left(1 / x^{K_{i_{m}}}\right) X_{B_{* \beta}^{m}}\left(x^{K^{i_{m}}}\right)$ est assurée par le fait que $B_{* \beta}^{m}$ ne comporte pas de terme $x^{I} B^{I}$ avec une composante de $I$ strictement négative. On montre qu'une solution $Z_{\beta+}^{m}$ de l'équation

$$
J^{2 m+1}\left(\left[\frac{1}{x^{K_{i_{m}}}} X_{\Pi^{m}}\left(x^{K_{i_{m}}}\right), Z_{\beta+}^{m}\right]\right)=\frac{1}{x^{K_{i_{m}}}} X_{B_{* \beta}^{m}}\left(x^{K_{i_{m}}}\right)
$$

est aussi une solution de (3.7). Une méthode utilisée par Bruno [2] et Stolovitch [8] dans des problèmes analogues, permets de majorer les solutions de (3.8).

\section{Démonstration du lemme 3.5}

Soit donc $\Pi^{m}$ une forme normale de Dufour-Wade, de partie quadratique $\Pi^{0}$, polynomiale de degré inférieur à $m+3$, satisfaisant à la condition $(\mathrm{C})$, et soit $B_{*}^{m} \in \widetilde{\mathcal{P}}^{m, 2 m}$ une somme de monômes non résonnants telle que la relation de compatibilité $J^{2 m+4}\left(\left[\Pi^{m}+\right.\right.$ $\left.\left.B_{*}^{m}, \Pi^{m}+B_{*}^{m}\right]\right)=0$ soit satisfaite. L'existence de solutions polynomiales $Z^{m} \in \mathcal{P}^{m, 2 m}$ de l'équation cohomologique $J^{2 m+m}\left(\left[\Pi^{m}, Z^{m}\right]\right)=B_{*}^{m}$ est conséquence du lemme 2.2 et a été montré par Dufour et Wade dans [6]. Insistons sur le fait que $Z^{m}$ n'est en général pas unique. 
4.1. Décomposition. Soit $\beta$ un poids non nul pour la représentation semi-simple $\rho^{m}$ de l'algèbre de Lie (abélienne) $\mathbb{S}$ dans l'espace vectoriel de champs de vecteurs formels $\mathcal{P}^{m, 2 m}$ (cf. section 3.1). On note $\mathcal{P}_{\beta}^{m, 2 m}$ l'espace de poids associé, c'est-à-dire $X \in \mathcal{P}^{m, 2 m}$ appartient à $\mathcal{P}_{\beta}^{m, 2 m}$ si $\left[S_{i}, X\right]=\beta_{i} X$, pour tout $1 \leq i \leq n$.

Définition 4.1. On note $\widetilde{\mathcal{P}}_{\beta}^{m, 2 m}$ l'espace de poids pour la représentation semi-simple $\tilde{\rho}^{m}$ (cf. section 3.1) : c'est l'espace des champs de bivecteurs $B=\sum_{m+1 \leq|I| \leq 2 m} x^{I} \Pi^{I}\left(\Pi^{I}\right.$ quadratique diagonal) appartenant à $\widehat{\mathcal{P}}^{m, 2 m}$, tel que $\mathcal{L}_{S_{i}}\left(x^{I}\right)=\beta_{i}, 1 \leq i \leq n$, pour tout multi-indice $I$ apparaissant effectivement dans la somme.

LEMME 4.1. Soit $\beta$ un poids non nul pour la représentation $\rho^{m}$. Alors la restriction $\grave{a} \mathcal{P}_{\beta}^{m, 2 m}$ de l'application $\mathcal{P}^{m, 2 m} \rightarrow \widetilde{\mathcal{P}}^{m, 2 m}, \quad X \rightarrow J^{2 m+2}\left(\left[\Pi^{m}, X\right]\right)$, est à valeurs dans $\widetilde{\mathcal{P}}_{\beta}^{m, 2 m}$.

Preuve. Du lemme 3.1 suit d'une part que si $X \in \mathcal{P}^{m, 2 m}$, alors $J^{2 m+2}\left(\left[\Pi^{m}, X\right]\right) \in \widetilde{\mathcal{P}}^{m, 2 m}$.

Soit $x^{K} \Pi^{K} \in \widehat{\mathcal{O}}^{S} \otimes \mathfrak{S}$ un terme de la forme normale tronqué $\Pi^{m}$, et soit $x^{I} Z^{I} \in$ $\mathcal{P}_{\beta}^{m, 2 m}$. Le fait que l'application du lemme 4.1 est à valeurs dans $\widetilde{\mathcal{P}}_{\beta}^{m, 2 m}$ suit de $\left[x^{I} Z^{I}, x^{K} \Pi^{K}\right] \in \widetilde{\mathcal{P}}_{\beta}^{m, 2 m}$. En effet, pour tout $1 \leq i \leq n$, on a $\mathcal{L}_{S_{i}}\left(x^{I}\right)=\beta_{i} x^{I}$ ainsi que $\mathcal{L}_{S_{i}}\left(x^{K}\right)=0$. Posons $\left[x^{I} Z^{I}, x^{K} \Pi^{K}\right]:=x^{J} \Pi^{J}$. Du lemme 3.1 suit $x^{J}=x^{I+K}$, et donc, pour tout $1 \leq i \leq n$, la relation $\mathcal{L}_{S_{i}}\left(x^{J}\right)=\beta_{i} x^{J}$.

Pour tout poids $\beta$ de la représentation $\tilde{\rho}^{m}$, notons $B_{* \beta}^{m}$ la projection de $B_{*}^{m}$ sur $\widetilde{\mathcal{P}}_{\beta}^{m, 2 m}$. On cherche les solutions $Z^{m}$ de l'équation cohomologique (3.6) sans termes résonnants, c'est-à-dire tel que la projection de $Z^{m}$ sur $\mathcal{P}_{0}^{m, 2 m}$ (l'espace de poids associé au poids nul) s'annule. On déduit du lemme précédent, que l'équation cohomologique (3.6) se décompose selon les différents poids $\beta \neq 0$ (pour les représentations $\rho^{m}$ et $\tilde{\rho}^{m}$ ) en des équations

$$
J^{2 m+2}\left(\left[\Pi^{m}, Z_{\beta}^{m}\right]\right)=B_{* \beta}^{m},
$$

où $Z_{\beta}^{m}$ appartient à $\mathcal{P}_{\beta}^{m, 2 m}$. Pour montrer le théorème 3.5 , il suffit donc de montrer la proposition suivante.

Proposition 4.1. Soit $\beta$ un poids non nul pour les représentations $\rho^{m}$ et $\tilde{\rho}^{m}$. Il existe des constantes $\eta>0, h>0$, déterminées par $\Pi^{0}$, telles que, pour tout entier $m>0$, il existe $Z_{\beta}^{m} \in \mathcal{P}_{\beta}^{m, 2 m}$ solution de l'équation (4.1), telle que pour tout $\left.\left.r \in\right] 1 / 2,1\right]$,

$$
\left\|\Pi^{m}-\Pi^{0}\right\|_{r}<\eta \text { et }\left\|D\left(\Pi^{m}-\Pi^{0}\right)\right\|_{r}<\eta \Longrightarrow\left\|Z_{\beta}^{m}\right\|_{r} \leq\left(1+\frac{m^{2} h}{|\beta|^{3}}\right)\left\|B_{* \beta}^{m}\right\|_{r} .
$$

4.2. Suppression des exposants négatifs. Cette section a pour objet de ramener la preuve de la proposition 4.1 au cas où $B_{* \beta}^{m}$ est une somme de monômes de la forme $x^{I} \Pi^{I}$, avec $\Pi^{I}$ champ de bivecteurs quadratique diagonal, et $I$ multi-indice ne comportant pas de composantes strictement négatives.

Soit $\widetilde{\mathcal{P}}$ l'espace vectoriel des champs de bivecteurs formels à 1 -jet nul, et désignons par $\widetilde{\mathcal{P}}^{+}$(respectivement $\widetilde{\mathcal{P}}^{-}$) le sous-espace engendré par les monômes $x^{I} P$, avec $P$ champ de bivecteurs quadratique diagonal, et $I \in \mathbb{N}^{n}$ (respectivement $I \in(\mathbb{N} \cup\{-1\})^{n}$ 
avec au moins une composante égale à -1 ). Clairement on a $\widetilde{\mathcal{P}}=\widetilde{\mathcal{P}}^{+} \oplus \widetilde{\mathcal{P}}^{-}$. On note $\operatorname{Pr}^{+}$ (respectivement $\operatorname{Pr}^{-}$) la projection sur $\widetilde{\mathcal{P}}^{+}$(respectivement $\widetilde{\mathcal{P}}^{-}$).

De même, soit $\mathcal{P}$ l'espace vectoriel des champs de vecteurs formels nuls à l'origine, et désignons par $\mathcal{P}^{+}$(respectivement $\mathcal{P}^{-}$) le sous espace engendré par les monômes $x^{I} L$, avec $L$ champ de vecteurs linéaire diagonal, et $I \in \mathbb{N}^{n}$ (respectivement $I \in(\mathbb{N} \cup\{-1\})^{n}$ avec exactement une composante égale à -1$)$. On a encore $\mathcal{P}=\mathcal{P}^{+} \oplus \mathcal{P}^{-}$.

Proposition 4.2. Soit $B_{* \beta}^{m}$ tel qu'en (4.1).

(i) Il existe un unique champ de vecteurs $Z_{\beta-}^{m} \in \mathcal{P}^{-} \cap \mathcal{P}_{\beta}^{m, 2 m}$ tel que

$$
\operatorname{Pr}^{-}\left(J^{2 m+1}\left[\Pi^{m}, Z_{\beta-}^{m}\right]\right)=B_{* \beta-}^{m},
$$

où $B_{* \beta-}^{m}:=\operatorname{Pr}^{-}\left(B_{* \beta-}^{m}\right)$.

(ii) Il existe des constantes positives $\eta_{1}$ et $k$, déterminées par $\Pi^{0}$, telles que pour tout $r \in] 1 / 2,1]$ on ait

$$
\left\|\Pi^{m}-\Pi^{0}\right\|_{r}<\eta_{1} \Longrightarrow\left\|Z_{\beta-}^{m}\right\|_{r} \leq \frac{m k}{|\beta|}\left\|B_{* \beta}^{m}\right\|_{r} .
$$

Preuve de proposition 4.2(i). Désignons par $\operatorname{Pr}_{\beta}^{m, 2 m}$ la projection sur $\widetilde{\mathcal{P}}_{\beta}^{m, 2 m}$. On a $B_{* \beta-}^{m}=\operatorname{Pr}^{-} \circ \operatorname{Pr}_{\beta}^{m, 2 m}(\Pi)$. Il suit donc de (2.5) et du lemme 4.1 qu'il suffit de montrer qu'il existe $Z_{-}^{m} \in \mathcal{P}^{-} \cap \mathcal{P}_{\beta}^{m, 2 m}$ unique avec

$$
\operatorname{Pr}^{-} \circ \operatorname{Pr}_{\beta}^{m, 2 m}\left(\exp \left(Z_{-}^{m}\right)^{*}(\Pi)\right)=0 .
$$

On procède par réccurence sur le degré $m+1<d \leq 2 m+2$. Supposons $J^{d+1}\left(\operatorname{Pr}^{-}\right.$。 $\left.\operatorname{Pr}_{\beta}^{m, 2 m}(\Pi)\right)=0$ et montrons qu'il existe $X \in \mathcal{P}_{\beta}^{m, 2 m}$ homogène de degré $d+1$ unique tel que $J^{d+2}\left(\operatorname{Pr}^{-} \circ \operatorname{Pr}_{\beta}^{m, 2 m}\left(\exp (X)^{*}(\Pi)\right)\right)=0$. Pour ceci, considérons le champ de bivecteurs $B$ et les champs de bivecteurs linéaires diagonaux $\Pi^{I},|I|=d-1$, tels que

$$
B:=\sum_{|I|=d-1} x^{I} \Pi^{I}:=J^{d+2}\left(\operatorname{Pr}^{-} \circ \operatorname{Pr}_{\beta}^{m, 2 m}(\Pi)\right)-J^{d+1}\left(\operatorname{Pr}^{-} \circ \operatorname{Pr}_{\beta}^{m, 2 m}(\Pi)\right) .
$$

Il suffit de montrer que pour tout $x^{I} \Pi^{I}$ tel que dans (4.4), il existe un unique champ de vecteurs linéaire diagonal $L^{I}$ avec $x^{I} L^{I} \in \mathcal{P}^{-} \cap \mathcal{P}_{\beta}^{m, 2 m}$ et tel que $x^{I} L^{I}$ est solution de l'équation

$$
\left[\Pi^{0}, x^{I} L^{I}\right]=x^{I} \Pi^{I} .
$$

Or, du fait qu'on a $\operatorname{Pr}^{-}\left(\Pi^{m}\right)=0$ (cf. la remarque après la définition 3.3), de l'identité de Jacobi $[\Pi, \Pi]=0$, du lemme 2.1 et du lemme 4.1 on déduit que pour tout champ de bivecteurs $x^{I} \Pi^{I}$ tel que dans (4.4) on a

$$
\left[\Pi^{0}, x^{I} \Pi^{I}\right]=0 .
$$

Par le lemme 2.2 on en déduit alors l'existence d'un champs de vecteurs linéaires diagonal $L^{I}$ tel que $x^{I} L$ est une solution holomorphe de (4.5). Comme $I$ contient exactement une composante strictement négative égale à -1 , l'unicité de $L^{I}$ suit de l'holomorphie de $x^{I} L^{I}$. L'appartenance $x^{I} L^{I} \in \mathcal{P}^{-} \cap \mathcal{P}_{\beta}^{m, 2 m}$ suit de $x^{I} \Pi^{I} \in \widetilde{\mathcal{P}}^{-} \cap$ $\widetilde{\mathcal{P}}_{\beta}^{m, 2 m}$. 
Avant de montrer la proposition 4.2(ii) introduisons quelques notations. Notons $\widetilde{\mathcal{P}}_{i}^{-}$, $1 \leq i \leq n$, le sous-espace de $\widetilde{\mathcal{P}}^{-}$engendré par les monômes de la forme $x^{I} \Pi^{I}$, avec $\Pi$ quadratique diagonal, et $I=\left(I_{1}, \ldots, I_{n}\right) \in(\mathbb{N} \cup\{-1\})^{n}$ tel que $I_{i}=-1$ et $I_{j} \geq 0$ pour $j \neq i$. De même, désignons par $\mathcal{P}_{i}^{-}$le sous-espace de $\mathcal{P}^{-}$engendré par les monômes de la forme $x^{I} Y_{i}$, avec $I=\left(I_{1}, \ldots, I_{n}\right) \in(\mathbb{N} \cup\{-1\})^{n}$ tel que $I_{i}=-1$ et $I_{j} \geq 0$ pour $j \neq i$. Désignons par $\widetilde{\mathcal{P}}_{i}^{+}$le sous-espace $\widetilde{\mathcal{P}}^{+}$engendré par les monômes de la forme $x^{I} P^{I}$, où $P^{I}$ quadratique diagonal, et $I=\left(I_{1}, \ldots, I_{n}\right) \in \mathbb{N}^{n}$ tel que $I_{i}=0$. On note $\operatorname{Pr}_{i}^{-}$la projection $B$ $\operatorname{sur} \widetilde{\mathcal{P}}_{i}^{-}, \operatorname{Pr}_{i}^{0}$ la projection sur $\widetilde{\mathcal{P}}_{i}^{+}$et $\operatorname{pr}_{i}^{-}$la projection sur $\mathcal{P}_{i}^{-}$.

Posons $B_{* \beta i}^{m}:=\operatorname{Pr}_{i}^{-}\left(B_{* \beta-}^{m}\right)\left(=\operatorname{Pr}_{i}^{-}\left(B_{* \beta}^{m}\right)\right)$.

De $\operatorname{Pr}^{-}\left(\Pi^{m}\right)=0$ il suit que la restriction à $\mathcal{P}_{i}^{-}$de l'application $X \rightarrow \operatorname{Pr}^{-}\left(\left[\Pi^{m}, X\right]\right)$ est à valeurs dans $\widetilde{\mathcal{P}}_{i}^{-}$. Par la conclusion de la proposition 4.2(i) et le lemme (2.1), on en déduit que l'équation (4.3) se décompose selon la composante des multi-indices qui vaut -1 . Plus précisément, on a $Z_{\beta-}^{m}=\sum_{i=1}^{n} Z_{i}^{m}$, où pour tout $1 \leq i \leq n$ le champ de vecteurs $Z_{i}^{m}:=\operatorname{pr}_{i}^{-}\left(Z_{\beta-}^{m}\right)$ est l'unique solution appartenant à $\mathcal{P}_{i}^{-} \cap \mathcal{P}_{\beta}^{m, 2 m}$ de l'équation

$$
J^{2 m+2}\left(\left[\Pi_{i}^{m}, Z_{i}^{m}\right]\right)=B_{* \beta i}^{m},
$$

avec $\Pi_{i}^{m}:=\operatorname{Pr}_{i}^{0}\left(\Pi^{m}\right)$. Par suite, la proposition 4.2(ii) est conséquence de la proposition suivante.

Proposition. (4.2 bis) Soit $1 \leq i_{0} \leq n$ fixé, et soit $Z_{i_{0}}^{m} \in \mathcal{P}_{i_{0}}^{-} \cap \mathcal{P}_{\beta}^{m, 2 m}$ l'unique solution de (4.6) pour $i=i_{0}$. Il existe deux constantes positives $\eta_{1}$ et $c$, déterminées par $\Pi^{0}$, telles que pour tout $r \in] 1 / 2,1]$

$$
\left\|\Pi_{i_{0}}^{m}-\Pi^{0}\right\|_{r}<\eta_{1} \Longrightarrow\left\|Z_{i_{0}}^{m}\right\|_{r} \leq \frac{m c}{|\beta|}\left\|B_{* \beta i_{0}}^{m}\right\|_{r} .
$$

Soit $\mathcal{N}:=\left\{J=\left(J_{1}, \ldots, J_{n}\right) \in \mathbb{N}^{n}: 0 \leq|J| \leq m, J_{i_{0}}=0, x^{J}\right.$ résonnant $\}$. Posons alors

$$
\Pi_{i_{0}}^{m}:=\sum_{J \in \mathcal{N}} x^{J} \Pi^{J}, \quad \Pi^{J}:=\frac{1}{2} \sum_{i \neq j} a_{i j}^{J} Y_{j} \wedge Y_{i}
$$

avec $a_{i j}^{J}=-a_{j i}^{J}$ (on a ainsi $\Pi^{(0, \ldots, 0)}=\Pi^{0}$ ). Par hypothèse $(\mathrm{C}), S_{i}^{J}:=\sum_{j=1}^{n} a_{i j}^{J} Y_{j} \in \mathbb{S}$. Soient donc $\beta_{i}^{J}, 1 \leq i \leq n, J \in \mathcal{N}$ définies par

$$
\left[S^{J}, X\right]=\beta_{i}^{J} X \quad \text { pour tout } X \in \mathcal{P}_{\beta}^{m, 2 m} .
$$

Comme $Z_{i_{0}}^{m} \in \mathcal{P}_{i_{0}}^{-}$on peut poser (pour $x_{i_{0}} \neq 0$ )

$$
Z_{i_{0}}^{m}:=g(x) Y_{i_{0}} .
$$

En développant le premier membre de (4.6) avec (2.1)-(2.4), et en utilisant que pour $J \in \mathcal{N}$ on a $\mathcal{L}_{Y_{i_{0}}}\left(x^{J}\right)=0$, on obtient le lemme suivant.

LEMme 4.2. Soient $\mathfrak{B}_{1}(x), \ldots, \mathfrak{B}_{n}(x)$ définies (pour $x_{i_{0}} \neq 0$ ) par

$$
\mathfrak{B}_{i}(x):=J^{2 m}\left(g(x) \sum_{J \in \mathcal{N}} x^{J} \beta_{i}^{J}\right), \quad 1 \leq i \leq n .
$$

Alors on a $B_{* \beta i}^{m}=\sum_{i=1}^{n} \mathfrak{B}_{i}(x) Y_{i} \wedge Y_{i_{0}}$.

La preuve du lemme 4.2 est donnée dans l'appendice. 
Lemme 4.3. Soit $P$ une structure de Poisson quadratique diagonale. Supposons $P \in \mathfrak{S}$, c'est-à-dire $P=(1 / 2) \sum_{i \neq j} b_{i j} Y_{i} \wedge Y_{j}$, avec $b_{i j}=-b_{j i}$ tel que $T_{i}:=\sum_{j=1}^{n} b_{i j} Y_{j} \in \mathbb{S}$. Fixons $1 \leq i_{0} \leq n$. Soit $I=\left(I_{1}, \ldots, I_{n}\right) \in(\mathbb{N} \cup\{-1\})^{n}$ tel que $|I| \geq 1$ et tel que $I_{i_{0}}=-1$, $I_{i} \geq 0$ pour $i \neq i_{0}$. Soient $\alpha_{i} \in \mathbb{C}, 1 \leq i \leq n$, tels que $\mathcal{L}_{T_{i}}\left(x^{I}\right)=\alpha_{i} x^{I}$ et posons $|\alpha|:=$ $\max _{1 \leq i \leq n}\left|\alpha_{i}\right|$. Alors

$$
n \max _{j \neq i_{0}}\left|\alpha_{i}\right| \geq \frac{1}{|I|}|\alpha| .
$$

Preuve. Si $\max _{1 \leq j \leq n}\left|\alpha_{j}\right|=\left|\alpha_{l}\right|$ avec $l \neq i_{0}$ il n'y a rien à démontrer. Supposons $\max _{1 \leq j \leq n}\left|\alpha_{j}\right|=\left|\alpha_{i_{0}}\right|$. Quitte à réordonner les coordonnées, on peut supposer $i_{0}=1$ et $\left|I_{n}\right|=\max _{1 \leq i \leq n}\left|I_{i}\right|$. Il s'agit de montrer $n \max _{j \neq i_{0}}\left|T_{i} . I\right| \geq(1 /|I|)\left|T_{1} . I\right|$. Exprimons, pour $2 \leq j \leq n-1$, les coefficients $b_{j n}$ en fonction des $\alpha_{j}$ et des coefficients $b_{j i}$ pour $i<n$ :

$$
\begin{aligned}
& \alpha_{j}:=T_{j} . I=-1 b_{j 1}+I_{2} b_{j 2}+\cdots+I_{n} b_{j n} \\
\Leftrightarrow & I_{n} b_{j n}=\alpha_{j}+b_{j 1}-I_{2} b_{j 2}-\cdots-I_{n-1} b_{j n-1} \\
\Leftrightarrow & b_{j n}=\frac{1}{I_{n}} \alpha_{j}+\frac{1}{I_{n}} b_{j 1}-\frac{I_{2}}{I_{n}} b_{j 2}-\cdots-\frac{I_{n-1}}{I_{n}} b_{j n-1} .
\end{aligned}
$$

Exprimons alors $\alpha_{n}:=T_{n} \cdot I=(-1)\left(-b_{1 n}\right)+I_{2}\left(-b_{2 n}\right)+\cdots+I_{n-1}\left(-b_{n-1 n}\right)$.

$$
\begin{aligned}
\alpha_{n}= & b_{1 n} \\
& -\frac{I_{2}}{I_{n}} b_{21}+\frac{I_{2}}{I_{n}}\left(\sum_{j=2}^{n-1} I_{j} b_{j 2}\right)-\frac{I_{2}}{I_{n}} \alpha_{2} \\
& \vdots \\
& -\frac{I_{n-1}}{I_{n}} b_{n-1,1}+\frac{I_{n-1}}{I_{n}}\left(\sum_{j=2}^{n-1} I_{j} b_{j, n-1}\right)-\frac{I_{n-1}}{I_{n}} \alpha_{n-1} .
\end{aligned}
$$

Par $b_{i j}=-b_{j i}$ les termes écrits entre parenthèses disparaissent et on obtient

$$
\alpha_{n}=\left(b_{1 n}+\frac{1}{I_{n}} \sum_{j=2}^{n-1} I_{j} b_{1 j}\right)-\sum_{j=2}^{n-1} \frac{I_{j}}{I_{n}} \alpha_{j}=\frac{1}{I_{n}} \alpha_{1}-\sum_{j=2}^{n-1} \frac{I_{j}}{I_{n}} \alpha_{j} .
$$

Avec $\left|I_{n}\right|=\max _{1 \leq i \leq n}\left|I_{i}\right|$ on en déduit $\alpha_{1} /|I| \leq \sum_{i \neq 1}\left|\alpha_{i}\right|$, i.e. $\alpha_{1} /|I| \leq n \max _{i \neq 1}\left|\alpha_{i}\right|$.

Corollaire 4.1. (Du lemme 4.3) Pour tout $i \leq i \leq n$ on $a$

$$
\left\|\mathfrak{B}_{i}\right\|_{r} \leq \frac{2 m n^{3}}{r^{2}}\left\|B_{* \beta_{i}}^{m}\right\|_{r} .
$$

Preuve du corollaire 4.1. Soit $\mathcal{B}:=\left\{I=\left(I_{1}, \ldots, I_{n}\right): x^{I} Y_{i_{0}} \in \mathcal{P}_{i_{0}}^{-} \cap \mathcal{P}_{\beta}^{m, 2 m}\right\}$. Posons $Z_{i_{0}}^{m}:=g(x) Y_{i_{0}}:=\sum_{L \in \mathcal{B}} c^{L} x^{L} Y_{i_{0}}$ ainsi que

$$
B_{* \beta i_{0}}^{m}:=\sum_{K \in \mathcal{B}} B^{K} \quad \text { et } \quad B^{K}:=\frac{1}{2} \sum_{i \neq j} x^{K} b_{i j}^{K} Y_{i} \wedge Y_{j},
$$


avec $b_{i j}^{K}=-b_{j i}^{K}$ (comme la $i_{0}$-ème coordonnée de $K$ est égale -1 on a $b_{i j}^{K} \neq 0$ uniquement si $i=i_{0}$ ou si $\left.j=i_{0}\right)$. Par le lemme 4.2,

$$
b_{i i_{0}}^{K}=\sum_{\substack{L+J=K \\ J \in \mathcal{N}, L \in \mathcal{B}}} c^{L} \beta_{i}^{J}:=\beta_{K, i}, \quad 1 \leq i \leq n \text { et } i \neq i_{0} .
$$

Pour majorer $\left\|\mathfrak{B}_{i_{0}}\right\|_{r}$ en fonction de $\left\|B_{* \beta_{i_{0}}}^{m}\right\|_{r}$ la difficulté provient du fait que le lemme 4.2 fournit pas de lien entre $\left(b_{i j}^{K}\right)_{i \neq j}$ et $\beta_{K, i_{0}}:=\sum_{\substack{L \in \mathcal{N}, L \in \mathcal{B} \\ J=K}} c^{L} \beta_{i_{0}}^{J}$. En appliquant le lemme 4.3 à la structure de Poisson quadratique diagonale $\sum_{\substack{L \in \mathcal{N}, L \in \mathcal{B} \\ L}} c^{L} \Pi^{J}$ (qui appartient à $\mathfrak{S}$ ), pour $I=K$ et $\alpha_{i}=\beta_{K, i}$ on obtient la majoration $\max _{1 \leq i \leq n}\left|\beta_{K, i}\right| \leq 2 m n \max _{i \neq i_{0}}\left|\beta_{K, i}\right|$. On en déduit

$$
\max _{1 \leq i \leq n}\left|\beta_{K, i}\right| \leq 2 m n \max _{i \neq i_{0}}\left|b_{i i_{0}}^{K}\right|=2 m n \max _{i \neq j}\left|b_{i j}^{K}\right| .
$$

Par le lemme 4.2,

$$
\left\|\mathfrak{B}_{i}\right\|_{r}=\left\|\sum_{K \in \mathcal{B}} x^{K} \beta_{K, i}\right\|_{r} \leq 2 m n\left\|\sum_{K \in \mathcal{B}} x^{K} \max _{i \neq j}\left|b_{i j}^{K}\right|\right\|_{r} .
$$

Par définition de la norme $\|\cdot\|_{r}$ (dans section 4), $\left\|B_{* \beta_{i_{0}}}^{m}\right\|_{r}=r^{2} \max _{i \neq j}\left\|\sum_{K \in \mathcal{B}} x^{K} b_{i j}^{K}\right\|_{r}$. D'où

$$
\left\|\sum_{K \in \mathcal{B}} x^{K} \max _{i \neq j}\left|b_{i j}^{K}\right|\right\|_{r} \leq \sum_{i \neq j}\left\|\sum_{K \in \mathcal{B}} x^{K} b_{i j}^{K}\right\|_{r} \leq \frac{n^{2}}{r^{2}}\left\|B_{* \beta_{i_{0}}}^{m}\right\|_{r} .
$$

Soit $1 \leq i_{m} \leq n$ tel que $|\beta|=\left|\beta_{i_{m}}\right|$, c'est-à-dire tel que $\max _{1 \leq i \leq n}\left|\beta_{i}\right|=\left|\beta_{i_{m}}\right|$.

LEMME 4.4. Il existe des constantes positives $\eta_{1}$ et $c^{\prime}$, déterminées par $\Pi^{0}$, telles que pour tout $r \in] 1 / 2,1]$ on ait

$$
\left\|\Pi_{i_{0}}^{m}-\Pi^{0}\right\|<\eta_{1} \Longrightarrow\|g(x)\|_{r} \leq \frac{m c^{\prime}}{\beta_{i_{m}}}\left\|\mathfrak{B}_{i_{m}}\right\|_{r} .
$$

La preuve de ce lemme est donnée en appendice.

Preuve de proposition 4.2 bis. La proposition 4.2 bis suit du corollaire 4.1 et du lemme 4.4.

4.3. Majoration dans le cas sans exposants négatifs. Soit $Z_{\beta-}^{m}$ le champ de vecteurs fourni par la proposition 4.2 et considérons l'équation

$$
J^{2 m+2}\left(\left[\Pi^{m}, Z_{\beta+}^{m}\right]\right)=\widetilde{B}_{* \beta}^{m}
$$

avec $\widetilde{B}_{* \beta}^{m}:=B_{* \beta}^{m}+J^{2 m+2}\left(\left[\Pi^{m}, Z_{\beta-}^{m}\right]\right)$. Par la proposition 4.2 on a $\widetilde{B}_{* \beta}^{m} \in \widetilde{\mathcal{P}}^{+}$. Signalons qu'on a pas l'unicité des solutions de (4.10) dans l'espace $\mathcal{P}_{\beta}^{m, 2 m}$. 
Proposition 4.3. Il existe des constantes positives $\eta_{2}$ et $l>0$, déterminées par $\Pi^{0}$, telles qu'il existe une solution $Z_{\beta+}^{m} \in \mathcal{P}_{\beta}^{m, 2 m}$ de (4.10) telle que pour tout $\left.\left.r \in\right] 1 / 2,1\right]$,

$$
\left\|\Pi^{m}-\Pi^{0}\right\|_{r}<\eta_{2} \text { et }\left\|D\left(\Pi^{m}-\Pi^{0}\right)\right\|_{r}<\eta_{2} \Longrightarrow\left\|Z_{\beta+}^{m}\right\|_{r} \leq\left(1+\frac{m l}{|\beta|^{2}}\right)\left\|\tilde{B}_{* \beta}^{m}\right\|_{r} .
$$

Posons

$$
\begin{aligned}
& \mathfrak{N}:=\left\{J \in \mathbb{N}^{n}: 1 \leq|J| \leq m, \mathcal{L}_{S_{i}}\left(x^{J}\right)=0,1 \leq i \leq n\right\} \\
& \mathfrak{B}:=\left\{I \in \mathbb{N}^{n}: m+1 \leq|I| \leq 2 m, \mathcal{L}_{S_{i}}\left(x^{I}\right)=\beta_{i} x^{I}, 1 \leq i \leq n\right\}
\end{aligned}
$$

ainsi que

$$
\Pi^{m}:=\Pi^{0}+\sum_{J \in \mathfrak{N}} x^{J} \Pi^{J} \quad \text { et } \quad \tilde{B}_{* \beta}^{m}:=\sum_{I \in \mathfrak{B}} x^{I} B^{I} .
$$

Pour $J \in \mathfrak{N}$ et $I \in \mathfrak{B}$ écrivons

$$
\begin{array}{rlrl}
\Pi^{J} & =\frac{1}{2} \sum_{i \neq j} a_{i j}^{J} Y_{i} \wedge Y_{j}, \quad S_{i}^{J}: & =\sum_{j=1}^{n} a_{i j} Y_{j} \\
\text { et } B^{I} & =\frac{1}{2} \sum_{i \neq j} b_{i j}^{I} Y_{i} \wedge Y_{j}, \quad B_{i}^{I}:=\sum_{j=1}^{n} b_{i j} Y_{j} .
\end{array}
$$

Notons. $K_{i}=(0, \ldots, 0,1,0, \ldots, 0)$ le multi-indice dont la $i$-ème composante est égale à 1 , et les autres égales à 0 . De (2.7) suit la relaion $X_{x^{I} \Pi^{I}}\left(x^{K_{i}}\right)=x^{I} x_{i} S_{i}^{I}$, d'où

$$
-\frac{1}{x^{K_{i}}} X_{\Pi^{m}}\left(x^{K_{i}}\right)=S_{i}+\sum_{J \in \mathfrak{N}} x^{J} S_{i}^{J} \quad \text { et } \quad-\frac{1}{x^{K_{i}}} X_{\tilde{B}_{* \beta}^{m}}\left(x^{K_{i}}\right)=\sum_{I \in \mathfrak{B}} x^{I} B_{i}^{I} .
$$

Remarque. L'holomorphie des champs de vecteurs (4.13) est garantie par l'absence d'exposants négatifs.

LEMME 4.5. Soit $1 \leq i \leq n$ tel que $\beta_{i} \neq 0$.

(i) Il existe un unique champ de vecteurs $Z_{i}$ appartenant à $\mathcal{P}_{\beta}^{m, 2 m}$ solution de

$$
J^{2 m+1}\left(\left[\frac{1}{x^{K_{i}}} X_{\Pi^{m}}\left(x^{K_{i}}\right), Z_{i}\right]\right)=\frac{1}{x^{K_{i}}} X_{\widetilde{B}_{* \beta}^{m}}\left(x^{K_{i}}\right) .
$$

(ii) $Z_{i}$ est également solution de (4.10).

Preuve du lemme 4.5. Par (4.13) le champ de vecteurs

$$
X:=\frac{1}{x^{K_{i}}} X_{\Pi^{m}+\widetilde{B}_{* \beta}^{m}}\left(x^{K_{i}}\right)=\frac{1}{x^{K_{i}}} X_{\Pi^{m}}\left(x^{K_{i}}\right)+\frac{1}{x^{K_{i}}} X_{\tilde{B}_{* \beta}^{m}}\left(x^{K_{i}}\right)
$$

est sous forme normale au degré $m+1$ au sens de Poincaré-Dulac. On peut alors normaliser $X$ au degré $2 m+1$ par une transformation de la forme $\mathrm{Id}+Z$, où $Z$ est un champ de vecteurs polynomial d'ordre $m+2$ et degré $2 m+1$ qui est solution de (4.14), i.e. solution de

$$
J^{2 m+1}\left(\left[S_{i}+\sum_{J \in \mathfrak{N}} x^{J} S_{i}^{J}, Z\right]\right)=\sum_{I \in \mathfrak{B}} x^{I} B_{i}^{I}
$$


(cf. (4.13)). En effet, pour $I \in \mathfrak{B}$ on a $\mathcal{L}_{S_{i}} x^{I}=x^{I} \beta_{i} \neq 0$ - il est alors facile de voir que (4.15) admet une unique solution $Z_{i}$ dans l'espace $\mathcal{P}_{\beta}^{m, 2 m}$. Reste à montrer que $Z_{i}$ est une solution de (4.10), ou de manière équivalente,

$$
\operatorname{Pr}_{\beta}^{m, 2 m}\left(\exp \left(Z_{i}\right)^{*}\left(\Pi^{m}+\tilde{B}_{\beta}^{m}\right)\right)=0,
$$

où on note $\operatorname{Pr}_{\beta}^{m, 2 m}$ la projection sur $\widetilde{\mathcal{P}}_{\beta}^{m, 2 m}$. Par (2.6) on a $\mathcal{L}_{\left[\left(1 / x^{K_{i}}\right) X_{\tilde{B}_{* \beta}^{m}}\left(x^{\left.\left.K_{i}\right)\right]}\right.\right.}\left(x^{K_{i}}\right)$

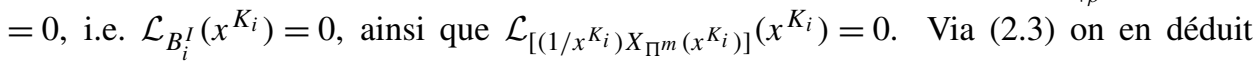
$\mathcal{L}_{Z_{i}}\left(x^{K_{i}}\right)=0$, i.e.

$$
\exp \left(Z_{i}\right)^{*}(X)=\frac{1}{x^{K_{i}}} X_{\exp \left(Z_{i}\right)^{*}\left(\Pi^{m}+\tilde{B}_{* \beta}^{m}\right)}\left(x^{K_{i}}\right)
$$

Or, puisque $\exp \left(Z_{i}\right)$ normalise $X$ au degré $2 m+1$ on a $\operatorname{pr}_{\beta}^{m, 2 m}(X)=0$ (on note $\operatorname{pr}_{\beta}^{m, 2 m}$ la projection sur $\mathcal{P}_{\beta}^{m, 2 m}$ ). D'après (4.17), ceci signifie que la relation (4.16) suit de l'implication

$$
\operatorname{Pr}_{\beta}^{m, 2 m}\left(\exp \left(Z_{i}\right)^{*}\left(\Pi^{m}+\tilde{B}_{\beta}^{m}\right)\right) \neq 0 \Longrightarrow \operatorname{pr}_{\beta}^{m, 2 m}(X) \neq 0 \text {. }
$$

Montrons donc (4.18). Supposons que $\operatorname{Pr}_{\beta}^{m, 2 m}\left(\exp \left(Z_{i}\right)^{*}\left(\Pi^{m}+\tilde{B}_{\beta}^{m}\right)\right)$ est non nul et admet un terme de plus petit degré $x^{I_{0}} \Pi^{I_{0}}$. Il s'agit de montrer $\left(1 / x^{K_{i}}\right) X_{x^{I_{0}} \Pi^{I_{0}}}\left(x^{K_{i}}\right) \neq 0$. De l'identité de Jacobi $[\Pi, \Pi]=0$ suit la relation $\left[\Pi^{0}, x^{I_{0}} \Pi^{I_{0}}\right]=0$. D'après le lemme 2.2, il existe un champ de vecteurs linéaire diagonal $D$ tel que $\left[\Pi^{0}, x^{I_{0}} D\right]=x^{I_{0}} \Pi^{I_{0}}$. Avec (2.9),

$$
x^{I_{0}} \Pi^{I_{0}}=D \wedge X_{\Pi^{0}}\left(x^{I_{0}}\right) .
$$

D'après (2.7), $X_{\Pi^{0}}\left(x^{I_{0}}\right)=-x^{I_{0}} \sum_{j=1}^{n} \beta_{j} Y_{j}$, d'où $c:=\mathcal{L}_{X_{\Pi^{0}}\left(x^{\left.I_{0}\right)}\right.}\left(x^{K_{i}}\right)=-x^{I_{0}} x_{i} \beta_{i} \neq 0$, puisque par hypothèse, $\beta_{i} \neq 0$. D'après (4.19) et (2.2),

$$
X_{x^{I_{0}} \Pi^{I_{0}}}\left(x^{K_{i}}\right)=c D-\mathcal{L}_{D}\left(x^{K_{i}}\right) X_{\Pi^{0}}\left(x^{I_{0}}\right) .
$$

Comme $x^{I_{0}} \Pi^{I_{0}} \neq 0$, (4.19) implique que les champs de vecteurs $X_{x^{I} \Pi^{I}}\left(x^{J}\right)$ et $D$ ne sont pas colinéaires. Il suit, avec $c \neq 0$, qu'on a bien $X_{x I_{0} \Pi^{I_{0}}}\left(x^{K_{i}}\right) \neq 0$.

Proposition 4.4. Soit $1 \leq i_{m} \leq n$ tel que $|\beta|=\left|\beta_{i_{m}}\right|$. Il existe deux constantes positives $\eta_{3}$ et $c$, determinées par $\Pi^{0}$, telles que l'unique solution $Z_{i_{m}}$ de l'équation (4.14) pour $i=i_{m}$ satisfait pour tout $\left.\left.r \in\right] 1 / 2,1\right]$,

$$
\begin{aligned}
& \left\|\frac{1}{x^{K_{i_{m}}}}\left[X_{\Pi^{m}}\left(x^{K_{i_{m}}}\right)-X_{\Pi^{0}}\left(x^{K_{i_{m}}}\right)\right]\right\|_{r}<\eta_{3} \text { et } \\
& \left\|D\left(\frac{1}{x^{K_{i_{m}}}}\left[X_{\Pi^{m}}\left(x^{K_{i_{m}}}\right)-X_{\Pi^{0}}\left(x^{K_{i_{m}}}\right)\right]\right)\right\|_{r}<\eta_{3} \\
& \Longrightarrow\left\|Z_{i_{m}}\right\|_{r} \leq\left(1+\frac{c}{|\beta|^{2}}\right)\left\|\frac{1}{x^{K_{i_{m}}}} X_{\widetilde{B}_{* \beta}^{m}}\left(x^{K_{i_{m}}}\right)\right\|_{r} .
\end{aligned}
$$

La proposition 4.4 est démontré dans la section suivante. 
Preuve de la proposition 4.3. Soit $1 \leq i_{m} \leq n$ tel que $|\beta|=\left|\beta_{i_{m}}\right|$. Par le lemme 4.5, l'unique solution $Z_{i_{m}}$ de l'équation (4.14) appartenant à l'espace $\mathcal{P}_{\beta}^{m, 2 m}$ est aussi une solution de (4.10). Or,

$$
\begin{aligned}
& \left\|\frac{1}{x^{K_{i}}} X_{\Pi^{m}}\left(x^{K_{i}}\right)-\frac{1}{x^{K_{i}}} X_{\Pi^{0}}\left(x^{K_{i}}\right)\right\|_{r} \leq \frac{1}{r}\left\|\Pi^{m}-\Pi^{0}\right\|_{r} \\
& \text { et }\left\|\frac{1}{x^{K_{i}}} X_{\tilde{B}_{* \beta}^{m}}\left(x^{K_{i}}\right)\right\|_{r} \leq \frac{1}{r}\left\|\tilde{B}_{* \beta}^{m}\right\|_{r} .
\end{aligned}
$$

Avec $Z_{\beta+}^{m}:=Z_{i_{m}}$, la proposition 4.3 suit de la proposition 4.4 via les majorations (4.21).

Preuve de la proposition 4.1. Par la proposition 4.2, il existe $\eta_{1}>0$ et $k>0$, determinées par $\Pi^{0}$, telles que pour tout $\left.\left.r \in\right] 1 / 2,1\right],\left\|\Pi^{m}-\Pi^{0}\right\|_{r}<\eta_{1} \Rightarrow\left\|Z_{\beta-}^{m}\right\|_{r} \leq$ $(m k /|\beta|)\left\|B_{* \beta}^{m}\right\|_{r}$. Comme $\Pi^{m}$ est de degré $\leq m+2$ et $Z_{\beta-}^{m}$ de degré $\leq 2 m+1$, il existe une constante $k^{\prime}>0$ telle que $\left\|\left[\Pi^{m}, Z_{\beta-}^{m}\right]\right\|_{r} \leq m k^{\prime}$. Si $\left\|\Pi^{m}-\Pi^{0}\right\|_{r}<\eta_{1}$ on a alors

$$
\begin{aligned}
\left\|\widetilde{B}_{* \beta}^{m}\right\|_{r} & \leq\left\|B_{* \beta}^{m}\right\|_{r}\left\|+J^{2 m+2}\left(\left[\Pi^{m}, Z_{\beta-}^{m}\right]\right)\right\|_{r} \leq\left\|B_{* \beta}^{m}\right\|_{r}+\frac{m^{2} k k^{\prime}}{|\beta|}\left\|B_{* \beta}^{m}\right\|_{r} \\
& \leq\left(1+\frac{m^{2} k k^{\prime}}{|\beta|}\right)\left\|B_{* \beta}^{m}\right\|_{r},
\end{aligned}
$$

car on avait posé $\widetilde{B}_{* \beta}^{m}=B_{* \beta}^{m}+J^{2 m+2}\left(\left[\Pi^{m}, Z_{\beta-}^{m}\right]\right)$. Le champ de vecteurs $Z_{\beta}^{m}:=Z_{\beta+}^{m}+$ $Z_{\beta-}^{m}$ satisfait (4.1). D'après (4.22) et la proposition 4.3 , avec $\eta:=\min \left(\eta_{1}, \eta_{2}\right)$, il existe $h>0$, determinée par $\Pi^{0}$, telle qu'on a (4.2) pour tout $\left.\left.r \in\right] 1 / 2,1\right]$.

4.4. Preuve de la proposition 4.4. On adapte les preuves données par Bruno et Stolovitch dans $[\mathbf{2}, \mathbf{8}]$. Soit $\beta$ un poids non nul pour la représentation $\rho^{m}$ et soit $\mathcal{P}_{\beta}^{m, 2 m}$ l'espace de poids associé. Pour alléger les écritures, posons

$$
N_{i_{m}}:=-\frac{1}{x^{K_{i}}} X_{\Pi^{m}}\left(x^{K_{i}}\right) \quad \text { et } \quad B_{i_{m}}:=-\frac{1}{x^{K_{i}}} X_{\tilde{B}_{* \beta}^{m}}\left(x^{K_{i}}\right) .
$$

On peut supposer que $S_{1}, \ldots, S_{l}$, avec $l \leq n$ constitue une base de l'algèbre de Lie $\mathbb{S}$, et on peut également supposer que l'on a $i_{m} \leq l$. On peut donc poser

$$
N_{i_{m}}=S_{i_{m}}+\sum_{J \in \mathfrak{N}} x^{J} S_{i_{m}}^{J}:=\sum_{j=1}^{l} h_{j}(x) S_{j} .
$$

Remarquons que les fonctions $h_{j}(x)$ appartiennent à l'ensemble $\hat{\theta}^{S}$ des intégrales premières formelles de l'algèbre $\mathbb{S}$. De plus, $h_{i_{m}}(0)=1$, et si $j \neq i_{m}$, alors on a $h_{j}(0)=0$. Avec ces notations, l'équation (4.14) devient $J^{2 m+1}\left(\left[N_{i_{m}}, Z_{i_{m}}\right]\right)=B_{i_{m}}$, où encore

$$
J^{2 m+1}\left(\sum_{j=1}^{l} h_{j}(x)\left[S_{j}, Z_{i_{m}}\right]+\sum_{j=1}^{l} \mathcal{L}_{Z_{i_{m}}} h_{j}(x) S_{j}\right)=B_{i_{m}} .
$$

Comme $Z_{i_{m}}$ appartient à l'espace de poids $\mathcal{P}_{\beta}^{m, 2 m},\left[S_{j}, Z_{i_{m}}\right]=\beta_{j} Z_{i_{m}}$ pour $1 \leq j \leq l$. La relation (4.25) peut alors s'écrire

$$
J^{2 m+1}\left(\beta_{i_{m}}\left(h_{i_{m}}(x)+\sum_{\substack{1 \leq j \leq l \\ j \neq i_{m}}} h_{j}(x) \frac{\beta_{j}}{\beta_{i_{m}}}\right) Z_{i_{m}}+\sum_{j=1}^{l} \mathcal{L}_{Z_{i_{m}}} h_{j}(x) S_{j}\right)=B_{i_{m}} .
$$


Rappelons que $i_{m}$ est un indice tel que $|\beta|:=\max _{1 \leq i \leq n}\left|\beta_{i}\right|=\left|\beta_{i_{m}}\right|$. Les $\beta_{j} / \beta_{i_{0}}$ sont donc bien définies et de valeur absolue majorée par un. Posons

$$
M(x):=\left(h_{i_{m}}(x)+\sum_{\substack{1 \leq j \leq l \\ j \neq i_{m}}} h_{j}(x) \frac{\beta_{j}}{\beta_{i_{m}}}\right) .
$$

La relation (4.26) devient $M(x) Z_{i_{m}}+\sum_{j=1}^{l} \mathcal{L}_{Z_{i_{m}}} h_{j}(x) S_{j}=B_{i_{m}}$, et si $M(x) \neq 0$,

$$
Z_{i_{m}}+\mathfrak{P}\left(Z_{i_{m}}\right)=\widetilde{B}_{i_{m}}
$$

où $\mathfrak{P}$ est l'opérateur

$$
\begin{aligned}
& \quad \mathcal{P}_{\beta}^{m, 2 m} \rightarrow \mathcal{P}_{\beta}^{m, 2 m}, \quad X \rightarrow J^{2 m+1}\left(\frac{1}{M(x) \beta_{i_{m}}} \sum_{1 \leq j \leq l} \mathcal{L}_{X} h_{j}(x) S_{j}\right), \\
& \text { et } \widetilde{B}_{i_{m}}:=J^{2 m+1}\left(\frac{1}{M(x) \beta_{i_{m}}} B_{i_{m}}\right) .
\end{aligned}
$$

L'operateur $\mathfrak{P}$ est nilpotent : on a $\mathfrak{P} \circ \mathfrak{P}=0$. En effet, l'opérateur $\mathfrak{P}$ est à valeurs dans le $\mathbb{C}$-espace vectoriel $\Sigma$ engendré par les champs de vecteurs de la forme $f(x) S_{i}$ pour $1 \leq$ $i \leq l$, où $f(x)$ désigne une fonction formelle. Or, rappelons que les fonctions $h_{j}(x)$ appartiennent à $\hat{\theta}^{S}$, les intégrales premières formelles de l'algèbre $\mathbb{S}$. Pour $X \in \Sigma$ on a donc $\mathfrak{P}(X)=0$.

En appliquant l'opérateur Id $-\mathfrak{P}$, la relation (4.27) devient

$$
Z_{i_{m}}=\widetilde{B}_{i_{m}}-\mathfrak{P}\left(\widetilde{B}_{i_{m}}\right) .
$$

LEMME 4.6. Il existe une constante positive $\eta_{3}$, determinée par $\Pi^{0}$, telle que, pour tout $1 / 2<r \leq 1$,

$$
\left\|N_{i_{m}}-S_{i_{0}}\right\|_{r} \leq \eta_{3} \Longrightarrow\|1 / M(x)\|_{r} \leq 2 .
$$

La preuve du lemme 4.6 est donnée dans l'appendice. Avec $\left|\beta_{i_{m}}\right|=|\beta|$ et le lemme 4.6 il existe alors $\eta_{3}^{\prime}>0$ tel que pour $1 / 2<r \leq 1$,

$$
\left\|N_{i_{m}}-S_{i_{m}}\right\|_{r}<\eta_{3} \Longrightarrow \widetilde{B}_{i_{m}} \leq \frac{2}{|\beta|}\left\|B_{i_{m}}\right\|_{r} .
$$

LEMME 4.7. Soit $\eta_{3}$ la constante du lemme 4.6. Il existe une constante positive $c^{\prime}$, determinée par $\Pi^{0}$, telle que pour tout $\left.\left.r \in\right] 1 / 2,1\right]$ et tout $X \in \mathcal{P}_{\beta}^{m, 2 m}$,

$$
\left\|N_{i_{m}}-S_{i_{m}}\right\|_{r} \leq \eta_{3} \text { et }\left\|D\left(N_{i_{m}}-S_{i_{m}}\right)\right\|_{r} \leq \eta_{3} \Longrightarrow\|\mathfrak{P}(X)\|_{r} \leq \frac{c^{\prime}}{|\beta|}\|X\|_{r} .
$$

Le lemme 4.7 est prouvé dans l'appendice. Si on a $\left\|N_{i_{m}}-S_{i_{m}}\right\|_{r} \leq \eta_{3}$ et $\| D\left(N_{i_{m}}-\right.$ $\left.S_{i_{m}}\right) \|_{r} \leq \eta_{3}$, on a d'après (4.28), (4.29) et le lemme 4.7,

$$
\begin{aligned}
\left\|Z_{i_{m}}\right\|_{r} & \leq\left\|\widetilde{B}_{i_{m}}\right\|_{r}+\left\|\mathfrak{P}\left(\widetilde{B}_{i_{m}}\right)\right\|_{r} \leq \frac{2}{|\beta|}\left\|B_{i_{m}}\right\|_{r}+\frac{2 c^{\prime}}{|\beta|^{2}}\left\|B_{i_{m}}\right\|_{r} \\
& \leq\left(1+\frac{c}{|\beta|^{2}}\right)\left\|B_{i_{m}}\right\|_{r},
\end{aligned}
$$

pour tout $r \in] 1 / 2,1]$ et une constante $c>0$ adéquate. Puisque $S_{i_{m}}=\left(1 / x^{K i_{m}}\right) X_{\Pi^{0}}\left(x^{K i_{m}}\right)$ on retrouve la proposition 4.4 . 


\section{Preuve du théorème de normalisation}

Champs de vecteurs, bivecteurs et transformations. Supposons la structure de Poisson holomorphe $\Pi$ du théorème 3.1 , normalisée au degré $2^{k_{0}+1}$ par une transformation $\Phi_{k_{0}}$ (holomorphe). Supposons qu'on ait une suite $\left(\Phi_{k}\right)_{k \geq k_{0}}:=\left(\mathrm{Id}+U_{k}\right)^{-1}$ de biholomorphismes normalisant $\Pi$ du degré $2^{k}$ à celui de $2^{k+1}$, avec $U_{k} \in \mathcal{P}^{2^{k}, 2^{k+1}}$. En particulier la transformation

$$
\Psi_{k}:=\Phi_{k-1} \circ \cdots \circ \Phi_{k_{0}}
$$

normalise $\Pi$ au degré $2^{k}+2$, c'est-à-dire on a

$$
\Psi_{k}^{*}(\Pi):=\Pi^{k}+B_{*}^{k}+B_{0}^{k}+R^{k},
$$

où :

$\Pi^{k}:=J^{\left(2^{k}+2\right)}\left(\Psi_{k}^{*}(\Pi)\right)$ désigne la forme normale tronquée au degré $2^{k}+2$ de $\Pi$ (on change de notation par rapport à la section précédente);

$B_{*}^{k}$ désigne la somme des termes non-résonnants de degré $d$, avec $2^{k}+3 \leq d \leq 2^{k+1}+2$; $B_{0}^{k}$ désigne la somme des termes résonnants de degré $d$, avec $2^{k}+3 \leq d \leq 2^{k+1}+2$;

$R^{k}$ désigne la série (convergente) constituée des termes de degrés strictement supérieurs à $2^{k+1}+2$.

Le champ de vecteurs $U_{k}$ est alors solution de

$$
J^{2^{k+1}+2}\left(\left[\Pi^{k}, U_{k}\right]\right)=B_{*}^{k} .
$$

Suite de rayons. Posons $\gamma_{k}=\left(m e / \omega_{k}^{3}\right)^{-1 / 2^{k}}$. La condition diophantienne $-\sum\left(\ln \omega_{k} / 2^{k}\right)<+\infty$ du théorème 3.1 implique la convergence du produit $\prod_{k=1}^{\infty} \gamma_{k}$. En effet,

$$
\left|\ln \left(\gamma_{k}\right)\right|=\left|\ln \left(\frac{m e}{\omega_{k}^{3}}\right)^{-1 / 2^{k}}\right|=\left|\frac{1}{2^{k}} \ln \left(\frac{m e}{\omega_{k}^{3}}\right)\right| \leq\left|\frac{1}{2^{k}} \ln (m e)\right|+3\left|\frac{1}{2^{k}} \ln \omega_{k}\right| .
$$

Définissons alors une suite de nombres réels positifs $\left(r_{k}\right)_{k \in \mathbb{N}}$ par récurrence sur $k$ en posant $r_{k+1}:=\gamma_{k}\left(2^{k}\right)^{-2 / 2^{k}} r_{k}$; la convergence du produit des nombres réels $\left(2^{k}\right)^{-2 / 2^{k}} r_{k}$, avec $k \geq 1$, permet de choisir $r_{0}$ de manière à ce $1 / 2<r_{k} \leq 1$ pour $k$ assez grand. La suite $\left(r_{k}\right)_{k \in \mathbb{N}}$ est strictement décroissante.

On a défini cette suite de rayons pour avoir la propriété suivante, utilisé dans la suite. Soit $\eta>0$ le nombre réel fourni par le lemme 3.5. Si on a

$$
\left\|\Pi^{k}-\Pi^{0}\right\|_{r}<\eta \quad \text { et } \quad\left\|D\left(\Pi^{k}-\Pi^{0}\right)\right\|_{r}<\eta,
$$

alors,

$$
\left\|B_{*}^{k}\right\|_{r_{k}} \leq 1 \Longrightarrow\left\|U^{k}\right\|_{r_{k}} \leq \gamma_{k}^{-2^{k}}
$$

Soient maintenant $\eta>0$ et $e>0$ les constantes fournis par le lemme 3.5 et supposons qu'on ait pour tout $k \geq k_{0},\left\|\Pi^{k}-\Pi^{0}\right\|_{r_{k}}<\eta$ et $\left\|D\left(\Pi^{k}-\Pi^{0}\right)\right\|_{r_{k}}<\eta$. D'après le lemme 3.5 on peut alors supposer que pour tout $\left.r \in] 1 / 2, r_{k}\right]$ on ait

$$
\left\|U_{k}\right\|_{r} \leq \frac{m e}{\omega_{m}^{3}}\left\|B_{*}^{k}\right\|_{r}
$$

(on rappelle $\omega_{m}=\inf \left\{\min _{1 \leq i \leq n}(|\alpha|): \alpha\right.$ poids non nul pour la représentation $\left.\rho^{m}\right\}$ ). 
Notation: Pour tout $r>0$, on note $D_{r}$ le polydisque (ouvert) de rayon $r$ de $\mathbb{C}^{n}$.

PROPOSITION 5.1. (De récurrence) Si $k_{0}$ est assez grand, alors on a les propriétés suivantes.

(i) Supposons qu'on a

$$
\begin{aligned}
& \text { (a) }\left\|\Pi^{k}-\Pi^{0}\right\|_{r_{k}} \leq \eta-\frac{4}{2^{k}}, \quad \text { (b) }\left\|D\left(\Pi^{k}-\Pi^{0}\right)\right\|_{r_{k}} \leq \eta-\frac{4}{2^{k}}, \\
& \text { et (c) }\left\|B_{*}^{k}+B_{0}^{k}+R^{k}\right\|_{r_{k}} \leq 1,
\end{aligned}
$$

alors on a

$$
\begin{aligned}
& \text { (a') }\left\|\Pi^{k+1}-\Pi^{0}\right\| \leq \eta-\frac{4}{2^{k+1}}, \quad\left(b^{\prime}\right)\left\|D\left(\Pi^{k+1}-\Pi^{0}\right)\right\|_{r_{k+1}} \leq \eta-\frac{4}{2^{k+1}}, \\
& \text { et (c') }\left\|B_{*}^{k+1}+B_{0}^{k+1}+R^{k+1}\right\|_{r_{k+1}} \leq 1 .
\end{aligned}
$$

(ii) Sous l'hypothèse de (i)(a), (b) et (c), on a $\left\|U_{k}\right\|_{r_{k+1}} \leq 1 /\left(2^{k}\right)^{2}$. De plus, la transformation $\left(\Phi_{k}\right)^{-1}:=\mathrm{Id}+U_{k}$ vérifie

$$
\left(\Phi_{k}\right)^{-1}\left(D_{r_{k+1}}\right) \subseteq D_{\left(2^{k}\right)^{-1 /\left(2^{k}\right)} r_{k}} \subseteq D_{r_{k}} .
$$

où $D_{r}$ désigne le polydisque (ouvert) de rayon $r$.

La preuve de la proposition 5.1 est donnée dans l'appendice. Elle admet comme conséquence l'existence d'une transformation convergente normalisant $\Pi$.

En effet, quitte à effectuer préalablement une transformation de la forme $t \mathrm{Id}, t>0$, on peut supposer sans perte de généralité, que la série définissant la structure de Poisson $\Pi$ converge sur le polydisque ouvert de rayon 1 . Soit $k_{0}$ l'entier fourni par la proposition 5.1. On choisi d'abord une transformation $\Phi_{k_{0}}$ de la forme $a \Phi_{0}$, avec $\Phi_{0}$ polynomiale normalisant $\Pi$ au degré $2^{k_{0}}$. On prend $a>0$ tel que $\Phi_{k_{0}}^{*}(\Pi)$ satisfait les hypothèses de récurence (i) (a), (b) et (c) de la proposition 5.1. Pour cela il suffit qu'on ait

$$
\begin{aligned}
a<\min \left[\left(\left\|\Phi_{0}^{*} \Pi\right\|_{r_{k_{0}}}\right)^{-1},\right. & \left(\left(\eta-\frac{4}{2^{k_{0}}}\right)\left\|\Pi^{k_{0}}-\Pi^{0}\right\|_{r_{k_{0}}}\right)^{-1 / d}, \\
& \left.\left(\left(\eta-\frac{4}{2^{k_{0}}}\right)\left\|D\left(\Pi^{k_{0}}-\Pi^{0}\right)\right\|_{r_{k_{0}}}\right)^{-1 /(d-1)}\right],
\end{aligned}
$$

où on note $d$ le degré minimal des termes non-quadratiques de $\Pi^{k_{0}}$.

La proposition 5.1 implique alors qu'il est effectivement possible de choisir une suite de transformations $\left(\Phi_{k}\right)_{k>k_{0}}$ normalisant $\Pi$ du degré $2^{k}+2$ à celui de $2^{k+1}+2$, de la manière décrite plus haut (i.e. $\Phi_{k}=\left(\mathrm{Id}+U_{k}\right)^{-1}$, où $U_{k} \in \mathcal{P}^{2^{k}, 2^{k+1}}$ est une solution de (5.1) fourni par le lemme 3.5). On pose

$$
\Psi:=\cdots \circ \Phi_{k_{0}+1} \circ \Phi_{k_{0}}=\lim _{k \rightarrow \infty} \Psi_{k}
$$

de la manière décrite plus haut. 
Pour établir le théorème 3.1, nous allons montrer l'holomorphie de $\Psi=\lim _{k \rightarrow \infty} \Psi_{k}$ sur le polydisque $D_{1 / 2}$ de rayon $1 / 2$. Plus précisément, on montrera que la suite d'applications

$$
f^{m}: x \rightarrow\left(\Psi_{k}\right)^{-1}(x)=\left(\operatorname{Id}+U^{k_{0}}\right) \circ \cdots \circ\left(\mathrm{Id}+U^{m}\right)(x)
$$

converge uniformément sur le polydisque $D_{1 / 2}$. Il en résulte l'holomorphie de $\lim _{k \rightarrow \infty}\left(\Psi_{k}\right)^{-1}$, et en conséquence, l'holomorphie de $\Psi=\lim _{k \rightarrow \infty} \Psi_{k}$ sur un voisinage de zéro.

Pour $x=\left(x_{1}, \ldots, x_{n}\right) \in \mathbb{C}^{n}$, notons $|x|:=\max _{1 \leq i \leq n}\left|x_{i}\right|$. Posons, pour $l \leq m$,

$$
f^{m, l}(x):=\left(\operatorname{Id}+U^{l}\right) \circ \cdots \circ\left(\operatorname{Id}+U^{m}\right)(x)
$$

Rappelons que pour tout entier $k$, on a $1 / 2<r_{k}<r_{k+1}<1$, et donc $D_{1 / 2} \subset D_{r_{k}} \subset D_{r_{k+1}}$. En itérant le point (ii) de la proposition 5.1 on obtient, pour $k_{0} \leq l \leq m$, la relation

$$
x \in D_{r_{m+1}} \Longrightarrow f^{m, l}(x) \in D_{r_{l}} .
$$

Soit $l_{0} \geq k_{0}$ un entier. Montrons par récurrence sur $m$ qu'on a, pour tout $m \geq l_{0}$, la relation

$$
x \in D_{r_{m+1}} \Longrightarrow\left|f^{m, l_{0}}(x)-x\right| \leq \sum_{k=l}^{m}\left\|U^{m+l_{0}-k}\right\|_{r_{m+l_{0}-k+1}} .
$$

Montrons (5.4) pour $m=l_{0}:$ si $x \in D_{r_{l_{0}+1}}$, on a

$$
\left|f^{l_{0}, l_{0}}(x)-x\right|=\left|U^{l_{0}}(x)\right| \leq\left\|U^{l_{0}}\right\|_{r_{l_{0}+1}} .
$$

Supposons qu'on a (5.4) pour l'entier $m$, et montrons (5.4) pour $m+1$. Soit $x \in D_{r_{m+2}}$. On a, par (5.3), que $\left(\mathrm{Id}+U^{m+1}\right)(x) \in D_{r_{m+1}}$. D'où par (5.4),

$$
\left|f^{m, l}\left(\operatorname{Id}+U^{m+1}\right)(x)-\left(\operatorname{Id}+U^{m+1}\right)(x)\right| \leq \sum_{k=l}^{m}\left\|U^{m+l-k}\right\|_{r_{m+l-k+1}} .
$$

Comme $f^{m+1, l}=f^{m, l} \circ\left(\mathrm{Id}+U^{m+1}\right)$ on a

$$
\left|f^{m+1, l}(x)-x\right| \leq \sum_{k=l}^{m+1}\left\|U^{m+L-k}\right\|_{r_{m+l-k+2}}
$$

par l'inégalité triangulaire.

Comme $D_{\frac{1}{2}} \subset D_{r_{k}}$ pour tout $k \geq 1$, la relation (5.4) implique en particulier $\mid f^{m}(x)-$ $x \mid \leq \sum_{k=k_{0}}^{m}\left\|U^{k}\right\|_{r_{k+1}}$ pour tout $x \in D_{\frac{1}{2}}$. En itérant la proposition 5.1, on obtient, pour $k \geq k_{0},\left\|U^{k}\right\|_{r_{k+1}} \leq 1 / 2^{k}$. La somme $\sum_{k=k_{0}}^{m}\left\|U^{k}\right\|_{r_{k+1}}$ converge donc pour $m \rightarrow \infty$. Il en résulte la convergence uniforme des l'applications $f^{m}$ sur le polydisque $D_{1 / 2}$, ce qui termine la preuve d'holomorphie de $\Psi$. 


\section{A. Appendice}

Preuve du Lemme 4.2. Avec (2.1)-(2.4) l'égalité (4.6) devient

$$
\begin{aligned}
B_{* \beta i_{0}}^{m}=J^{2 m+2}\left(\left[\Pi_{i_{0}}^{m}, Z_{i_{0}}^{m}\right]\right) & =J^{2 m+2}\left(\left[\Pi_{i_{0}}^{m}, g(x) Y_{i_{0}}\right]\right) \\
& =-J^{2 m+2}\left(X_{\Pi_{i_{0}}^{m}}(g(x)) \wedge Y_{i_{0}}-\left[\Pi_{i_{0}}^{m}, Y_{i_{0}}\right]\right) \\
& =-J^{2 m+2}\left(\sum_{J \in \mathcal{N}} x^{J} X_{\Pi^{J}}(g(x)) \wedge Y_{i_{0}}-\left[\sum_{J \in \mathcal{N}} x^{J} \Pi^{J}, Y_{i_{0}}\right]\right) .
\end{aligned}
$$

De $\left[\Pi^{J}, Y_{i_{0}}\right]=0$ il suit $\left[\sum_{J \in \mathcal{N}} x^{J} \Pi^{J}, Y_{i_{0}}\right]=\sum_{J \in \mathcal{N}} \mathcal{L}_{Y_{i_{0}}}\left(x^{J}\right) \Pi^{J}=0 . \quad$ D' autre part $-X_{\Pi^{J}}(g(x))=\sum_{i=1}^{n} \mathcal{L}_{S_{i}^{J}}(g(x)) Y_{i}=\sum_{i=1}^{n} \beta_{i}^{J} g(x) Y_{i}$, puisque $Z_{i_{0}}^{m}=g(x) Y_{i_{0}} \in \mathcal{P}_{\beta}^{m, 2 m}$. D'où

$$
B_{* \beta i_{0}}^{m}=-J^{2 m+2}\left(\sum_{J \in \mathcal{N}} x^{J} X_{\Pi^{J}}(g(x)) \wedge Y_{i_{0}}\right)=J^{2 m+2}\left(\sum_{i=1}^{n} g(x) \sum_{J \in \mathcal{N}} x^{J} \beta_{i}^{J} Y_{i} \wedge Y_{i_{0}}\right)
$$

Preuve du lemme 4.4. Sans perte de généralité on peut supposer que $S_{1}, \ldots, S_{l}$ est une base de $\mathbb{S}$ tel que $1 \leq i_{m} \leq l \leq n$. Posons

$$
\sum_{J \in \mathcal{N}} x^{J} S_{i}^{J}:=\sum_{j=1}^{l} h_{i j}(x) S_{j}
$$

Comme $S_{i}^{0}=S_{i}$ pour $1 \leq i \leq n$, on a $h_{i i}(0)=1$ pour $1 \leq i \leq n$. Alors

$$
J^{2 m}\left(g(x) \sum_{j=1}^{l} h_{i_{m} j}(x) \beta_{j}\right)=\mathfrak{B}_{i_{m}} .
$$

Comme $\max _{1 \leq i \leq l}\left|\beta_{i}\right|=\left|\beta_{i_{m}}\right|>0$, on peut écrire

$$
J^{2 m}\left(g(x) \beta_{i_{m}}\left[h_{i_{m} i_{m}}(x)+\sum_{\substack{1 \leq j \leq l \\ j \neq i_{0}}} h_{i_{m} l}(x) \frac{\beta_{j}}{\beta_{i_{m}}}\right]\right)=\mathfrak{B}_{i_{m}} .
$$

Soit $M(x):=\left[h_{i_{m} i_{m}}(x)+\sum_{\substack{1 \leq j \leq l \\ j \neq i_{0}}} h_{i_{m} j}(x)\left(\beta_{j} / \beta_{i_{m}}\right)\right]$. Puisque $g$ est un polynôme de degré $\leq 2 m$ on a, $\operatorname{si} M(x) \neq 0$,

$$
g(x)=J^{2 m}\left(\frac{1}{\beta_{i_{m}} M(x)} \mathfrak{B}_{i_{m}}\right) .
$$

D'après le lemme A.1 (plus bas) il existe $\eta_{1}>0$, indépendant de $m$, tel que pour tout $r \in] 1 / 2,1],\left\|\Pi_{i_{m}}^{m}-\Pi^{0}\right\|_{r} \leq \eta_{1} \Longrightarrow\|1 / M(x)\|_{r} \leq 2$.

Lemme A.1. Soit $M(x)$ tel que dans la preuve du lemme 4.4. Il existe une constante positive $\eta_{1}>0$, déterminée par $\Pi^{0}$, telle que pour tout $\left.\left.r \in\right] 1 / 2,1\right],\left\|\Pi_{i_{0}}^{m}-\Pi^{0}\right\|_{r} \leq$ $\eta_{1} \Longrightarrow\|1 / M(x)\|_{r} \leq 2$.

Preuve du lemme A.1. On emprunte la preuve de Stolovitch [8, p. 185]. Ecrivons

$$
\sum_{J \in \mathcal{N}} x^{J} S^{J}=\sum_{j=1}^{l} h_{i j}(x) S_{j}:=\sum_{k=1}^{n} g_{i k}(x),
$$


où on on a posé $g_{i k}(x):=\sum_{j=1}^{l} h_{i j}(x) a_{j k}$. La définition des $g_{i k}(x)$ peut s'écrire sous forme matricielle

$$
\left(\begin{array}{c}
g_{i 1}(x) \\
\vdots \\
g_{i n}(x)
\end{array}\right)=\left(\begin{array}{ccc}
a_{11} & \cdots & a_{l 1} \\
\vdots & & \vdots \\
a_{1 l} & \cdots & a_{l n}
\end{array}\right)\left(\begin{array}{c}
h_{i 1}(x) \\
\vdots \\
h_{i l}(x)
\end{array}\right) .
$$

Remarquons que les coefficients de la matrice $L:=\left(a_{i j}\right)_{1 \leq i \leq n, 1 \leq j \leq l}$ proviennent de la partie quadratique $\Pi^{0}$. Comme par hypothèse, les $S_{j}, 1 \leq j \leq l$, sont linéairement indépendants, la matrice $L$ est de rang $l$. On peut supposer, sans perte de généralité, que $L:=\left(a_{i j}\right)_{1 \leq i \leq n, 1 \leq j \leq l}$ est inversible d'inverse $L^{-1}:=\left(\tilde{a}_{i j}\right)_{\substack{1 \leq i \leq n \\ 1 \leq j \leq l}}$. On peut donc écrire pour $1 \leq j \leq n$,

$$
h_{i j}(x)-h_{i j}(0)=\sum_{k=1}^{n} \tilde{a}_{j k}\left(g_{i k}(x)-g_{i k}(0)\right) .
$$

Par la définition des $S_{i}^{J}$ et (4.7),

$$
\left\|\sum_{J \in \mathcal{N}} x^{J} S^{J}\right\|_{r} \leq 1 / r\left\|\Pi_{i_{0}}^{m}\right\|_{r}, \quad\left\|\left(\sum_{J \in \mathcal{N}} x^{J} S^{J}\right)-S_{i}\right\|_{r} \leq 1 / r\left\|\Pi_{i_{0}}^{m}\right\|_{r} .
$$

(On divise par $r$ parce qu'il manque les $Y_{i}:=x_{i}\left(\partial / \partial x_{i}\right)$ du deuxième facteur du produit exterieur.) La relation (A.3) implique

$$
\left\|g_{i k}(x)-g_{i k}(0)\right\| \leq 1 / r\left\|\left(\sum_{J \in \mathcal{N}} x^{J} S^{J}\right)-S_{i}\right\|_{r} .
$$

D'où $\left\|g_{i k}(x)-g_{i k}(0)\right\| \leq 1 / r^{2}\left\|\Pi_{i_{0}}^{m}\right\|_{r}$. Avec (A.4) on en déduit

$$
\left\|h_{i j}(x)-h_{i j}(0)\right\|_{r} \leq \frac{n}{r^{2}}\left\|L^{-1}\right\|\left\|\Pi_{i_{0}}^{m}-\Pi^{0}\right\|_{r},
$$

où on a posé $\left\|L^{-1}\right\|:=\max _{j, k}\left|\tilde{a}_{j k}\right|$. Comme $|\beta|=\max _{1 \leq i \leq n}\left|\beta_{i}\right|=\left|\beta_{i_{m}}\right|$ on a

$$
\left|\beta_{j} / \beta_{i_{m}}\right| \leq 1 \quad \text { pour } 1 \leq j \leq l .
$$

Or, $h_{i_{m} i_{m}}(0)=1$ et $h_{i_{m} j}=0$ si $j \neq i_{m}$. On peut donc poser $M(x)=1+H(x)$, où $H(x):=$ $\sum_{j=1}^{n}\left(h_{i_{m} j}(x)-h_{i_{m} j}(0)\right)\left(\beta_{j} / \beta_{i_{m}}\right)$ est une fonction polynomiale sans partie constante. Puisque $1 / M=1 /(1+H)=1+\sum_{m \geq 1}(-1)^{m} H^{m}$ on a $\|1 / M\|_{r} \leq 1+\sum_{m \geq 1}\left(\|H\|_{r}\right)^{m}$. De plus, par (A.6),

$$
\|H(x)\|_{r} \leq\left\|\sum_{i=j}^{n} h_{i_{m} j}(x)-h_{i_{m} j}(0)\right\|_{r} .
$$

Donc pour avoir $\|1 / M(x)\|_{r} \leq 2$ il suffit qu'on a $\|H(x)\|_{r} \leq 1 / 2$, et ceci est assuré si

$$
\left\|\sum_{i=j}^{n} h_{i_{m} j}(x)-h_{i_{m} j}(0)\right\|_{r} \leq 1 / 2 \text {. }
$$

D'après (A.5), ceci est assuré si on a la relation $\left\|\Pi_{i_{0}}^{m}-\Pi^{0}\right\|_{r} \leq 1 /\left(2 r^{2} n^{2}\left\|L^{-1}\right\|\right)$, où $\left\|L^{-1}\right\|=\max _{i, j}\left|\tilde{a}_{i j}\right|$. Le nombre réel $\eta_{1}:=1 /\left(2 r^{2} n^{2}\left\|L^{-1}\right\|\right)$ satisfait donc les demandes du lemme A.1. 
Preuve du lemme 4.6. On emprunte la preuve de Stolovitch [8, p. 185]. On a

$$
N_{i_{m}}=\sum_{\substack{1 \leq j \leq l \\ 1 \leq k \leq n}} h_{j}(x) a_{j k} Y_{k}:=\sum_{k=1}^{n} g_{k}(x) Y_{k},
$$

où on a posé $g_{k}(x):=\sum_{j=1}^{l} h_{j}(x) a_{j k}$. Matriciellement ceci s'écrit

$$
\left(\begin{array}{c}
g_{1} \\
\vdots \\
g_{n}
\end{array}\right)=\left(\begin{array}{ccc}
a_{11} & \cdots & a_{l 1} \\
\vdots & & \vdots \\
a_{1 n} & \cdots & a_{l n}
\end{array}\right)\left(\begin{array}{c}
h_{1}(x) \\
\vdots \\
h_{l}(x)
\end{array}\right)
$$

avec $l \leq n$ (les $a_{i j}$ proviennent de la partie quadratique $\Pi^{0}$ ). Comme les champs de vecteurs linéaires diagonaux $S_{1}, \ldots, S_{l}$ sont linéairement indépendants sur $\mathbb{C}$, la matrice $L:=\left(a_{j k}\right)_{1 \leq j \leq l, 1 \leq k \leq n}$ est de rang $l$. Sans perte de généralité, on peut supposer que la matrice $L$ est inversible d'inverse $L^{-1}:=\left(\tilde{a}_{j k}\right)_{1 \leq i \leq n, 1 \leq j \leq n}$. On peut donc écrire pour $1 \leq j \leq n$,

$$
h_{j}(x)-h_{j}(0)=\sum_{k=1}^{n} \tilde{a}_{j k}\left(g_{k}(x)-g_{k}(0)\right) .
$$

Or, comme $S_{i_{m}}$ est partie linéaire du champ de vecteurs $N_{i_{m}}$, on a, d'après (A.8), $N_{i_{m}}(x)-S_{i_{m}}=\sum_{k=1}^{n}\left(g_{k}(x)-g_{k}(0)\right) Y_{k}$. Cette dernière relation implique

$$
\left\|g_{k}(x)-g_{k}(0)\right\|_{r} \leq 1 / r\left\|N_{i_{m}}-S_{i_{m}}\right\|_{r}
$$

(on divise par $r$ puisque $Y_{i}:=x_{i}\left(\partial / \partial x_{i}\right)$ ). Avec (A.9), on en déduit

$$
\left\|h_{j}(x)-h_{j}(0)\right\|_{r} \leq n / r\left\|L^{-1}\right\|\left\|N_{i_{m}}-S_{i_{m}}\right\|_{r},
$$

où on a posé $\left\|L^{-1}\right\|:=\max _{j, k}\left|\tilde{a}_{j k}\right|$. Posons $M(x)=1+H(x)$, où $H(x):=$ $\sum_{j=1}^{n}\left(h_{j}(x)-h_{j}(0)\right)\left(\beta_{j} / \beta_{i_{m}}\right)$ est une fonction polynomiale sans partie constante. Comme $1 / M=1+\sum_{m \geq 1}(-1)^{m} H^{m},\|1 / M(x)\|_{r} \leq 2$ est assuré si $\|H(x)\|_{r} \leq 1 / 2$. Or, $\operatorname{par}\left|\beta_{j} / \beta_{i_{0}}\right|<1$,

$$
\|H(x)\|_{r} \leq\left\|\sum_{j=1}^{n} h_{j}(x)-h_{j}(0)\right\|_{r} .
$$

Pour avoir $\|1 / M(x)\|_{r} \leq 2$, il suffit donc $\left\|\sum_{i=j}^{n} h_{j}(x)-h_{j}(0)\right\|_{r} \leq 1 / 2$. Pour cela, d'après (A.10), il suffit qu'on a $\left\|N_{i_{m}}-S_{i_{m}}\right\|_{r} \leq r /\left(2 n\left\|L^{-1}\right\|\right)$.

Preuve du lemme 4.7. Soit $f=\sum_{I \in \mathbb{N}^{n}} f_{I} x^{I}$ une série formelle. Posons $\bar{f}:=$ $\sum_{I \in \mathbb{N}^{n}}\left|f_{I}\right| x^{I}$. Si $f$ et $g$ sont deux séries formelles on note $f \prec g$ si on a $\left|f_{I}\right|<\left|g_{I}\right|$ pour tout $I \in \mathbb{N}^{n}$. Pour tout $1 \leq k \leq n$ on peut écrire

$$
\frac{\partial x_{k} \overline{\left(g_{k}(x)-g_{k}(0)\right)}}{\partial x_{p}}=\delta_{k p} \overline{\left(g_{k}(x)-g_{k}(0)\right)}+x_{k} \frac{\partial \overline{\left(g_{k}(x)-g_{k}(0)\right)}}{\partial x_{p}},
$$

ou bien

$$
x_{k} \frac{\partial \overline{\left(g_{k}(x)-g_{k}(0)\right)}}{\partial x_{p}}=\frac{\partial x_{k} \overline{\left(g_{k}(x)-g_{k}(0)\right)}}{\partial x_{p}}-\delta_{k p} \overline{\left(g_{k}(x)-g_{k}(0)\right)} \text {. }
$$


Mais, comme $x_{k}\left(\partial \overline{\left(g_{k}(x)-g_{k}(0)\right)} / \partial x_{p}\right), \partial x_{k} \overline{\left(g_{k}(x)-g_{k}(0)\right)} / \partial x_{p}$ et $\overline{\left(g_{k}(x)-g_{k}(0)\right)}$ sont des séries formelles à coefficients non négatives, on a

$$
x_{p} \frac{\partial \overline{\left(g_{k}(x)-g_{k}(0)\right)}}{\partial x_{k}} \prec \frac{\partial x_{k} \overline{\left(g_{k}(x)-g_{k}(0)\right)}}{\partial x_{p}} .
$$

Comme $\frac{1}{2}<r$,

$$
\begin{aligned}
\left\|\frac{\partial \overline{\left(g_{k}(x)-g_{k}(0)\right)}}{\partial x_{p}}\right\|_{r} & \leq 2 r\left\|\frac{\partial \overline{\left(g_{k}(x)-g_{k}(0)\right)}}{\partial x_{p}}\right\|_{r} \\
& \leq 2\left\|x_{k} \frac{\partial \overline{\left(g_{k}(x)-g_{k}(0)\right)}}{\partial x_{p}}\right\|_{r} \\
& \leq 2\left\|\frac{\partial x_{k} \overline{\left(g_{k}(x)-g_{k}(0)\right)}}{\partial x_{p}}\right\|_{r} .
\end{aligned}
$$

Il suit que, pour tout $1 \leq j \leq n$,

$$
\begin{aligned}
\left\|\frac{\partial \overline{\left(h_{j}(x)-h_{j}(0)\right)}}{\partial x_{p}}\right\|_{r} & \leq \sum_{k=1}^{n}\left|\tilde{a}_{j k}\right|\left\|\frac{\partial \overline{\left(g_{k}(x)-g_{k}(0)\right)}}{\partial x_{p}}\right\|_{r} \\
& \leq 2 \sum_{k=1}^{n}\left|\tilde{a}_{j k}\right|\left\|\frac{\partial x_{k} \overline{\left(g_{k}(x)-g_{k}(0)\right)}}{\partial x_{p}}\right\|_{r} \\
& \leq 2 n\left\|L^{-1}\right\|\left\|D\left(N_{i_{m}}-S_{i_{m}}\right)\right\|_{r} .
\end{aligned}
$$

Avec $\left\|\partial \overline{\left(h_{j}(x)-h_{j}(0)\right)} / \partial x_{p}\right\|_{r}=\left\|\partial h_{j}(x) / \partial x_{p}\right\|_{r}$ on a pour tout $X \in \mathcal{P}_{\beta}^{m, 2 m}$,

$$
\left\|\mathcal{L}_{X} h_{j}(x) S_{j}\right\|_{r} \leq\|X\|_{r}\left\|D\left(N_{i_{m}}-S_{i_{m}}\right)\right\|_{r}\left(2 n^{2}\left\|L^{-1}\right\|\left\|S_{j}\right\|_{r}\right) .
$$

Rappelons $\mathfrak{P}(X)=(1 / M(x)) \sum_{j=1}^{n} \mathcal{L}_{X} h_{j}(x) S_{j}$. Soit $\eta_{3}$ le nombre réel fournit par le lemme 4.6. Si $r \in] 1 / 2$, 1] et si $\left\|\left(N_{i_{m}}-S_{i_{m}}\right)\right\|_{r} \leq \eta_{3}$, le lemme 4.6 affirme $\|1 / M(x)\|_{r}$ $\leq 2$. D'où

$$
\|\mathfrak{P}(X)\|_{r} \leq\|X\|_{r} \frac{1}{\left|\beta_{i_{m}}\right|}\left\|D\left(N_{i_{m}}-S_{i_{m}}\right)\right\|_{r} 4 n^{3}\left\|L^{-1}\right\|\left(\max _{1 \leq j \leq n}\left\|S_{j}\right\|_{r}\right) .
$$

Rappelons encore $\left\|\beta_{i_{m}}\right\|=\|\beta\|$. Le lemme 4.7 s'obtient avec

$$
c^{\prime}=\eta_{3} 4 n^{3}\left\|L^{-1}\right\|\left(\max _{1 \leq j \leq n}\left\|S_{j}\right\|_{r}\right) .
$$

Démonstration de la proposition 5.1.

Lemmes préliminaires. La démonstration de la proposition 5.1 repose sur le lemme suivant.

LEMME A.2.

(i) Soit P une fonction holomorphe (respectivement un champ de vecteurs holomorphe, un champ de bivecteurs holomorphe) nul à l'origine et d'ordre $2^{k}$ (c'est-à-dire que le terme de plus petit degré est de degré $\left.2^{k}\right)$. Soient $a, r>0$ des nombres réels, et supposons $a<1$. Alors on a

$$
\|P\|_{a r} \leq a^{\left(2^{k}\right)}\|P\|_{r}
$$


En particulier,

$$
\begin{aligned}
& \text { (1) }\|P\|_{r_{k}} \leq 1 \Rightarrow\|P\|_{r_{k+1}} \leq 1 /\left(2^{k}\right)^{2}\|P\|_{r_{k}}, \\
& \text { et (2) }\|P\|_{\left(2^{k}\right)^{-1 /\left(2^{k}\right)} r_{k}} \leq 1 / 2^{k}\|P\|_{r_{k}} .
\end{aligned}
$$

(ii) Soit $0<r<1$ fixé. Soit $P$ un champ de vecteurs, et supposons $\|P\|_{\left(2^{k}\right)^{-1 /\left(2^{k}\right) r}} \leq$ $1 /\left(2^{k}\right)^{2}$. Alors pour $k$ assez grand,

$$
(\mathrm{Id}+P)\left(D_{\left(2^{k}\right)^{-1 /\left(2^{k}\right) r}}\right) \subseteq D_{r} .
$$

Preuve du lemme A.2. Preuve de (i) : pour (1) $a=\gamma_{k}\left(2^{k}\right)^{-1 /\left(2^{k}\right)} \leq\left(2^{k}\right)^{-1 /\left(2^{k}\right)}$ convient ; pour (2) $a=\left(2^{k}\right)^{-1 /\left(2^{k}\right)}$ convient. Pour (ii) il suffit de montrer $\left(2^{k}\right)^{-1 /\left(2^{k}\right)} r+1 /\left(2^{k}\right)^{2}<r$, c'est-à-dire, $1 /\left(2^{k}\right)^{2}<\left(1-\left(2^{k}\right)^{-1 /\left(2^{k}\right)}\right) r$. Or, si $k$ est assez grand, on a $\left|\left(2^{k}\right)^{-1 / 2^{k}}\right|>$ $1-\left(1 / 2^{k}\right) \ln \left(2^{k}\right)$. Donc il suffit que pour $k$ assez grand $1 /\left(2^{k}\right)^{2}<\left(1 / 2^{k}\right) \ln \left(2^{k}\right)$, ce qui est vrai.

La suite $\left(\gamma_{k}\right)_{k \geq 1}$, a été choisie, pour obtenir, à partir du lemme A.2, les deux lemmes suivants sur lesquels repose la démonstration de la proposition 5.1.

Lemme A.3. Soit $P$ un champ de vecteurs holomorphe. Supposons $\|P\|_{r_{k+1}} \leq 1 /\left(2^{k}\right)^{2}$. Pour $k$ assez grand, le difféomorphisme $\mathrm{Id}+P$ verifie la relation

$$
(\operatorname{Id}+P)\left(D_{r_{k+1}}\right) \subseteq D_{\left(\left(2^{k}\right)^{-1 / 2^{k}}\right) r_{k}} .
$$

Preuve du lemme A.3. Puisqu'on a $\left|\gamma_{k}\right| \leq 1$, ce lemme est un corollaire de la partie (ii) du lemme A.2.

\section{LEMME A.4.}

(i) Soit $P$ un champ de vecteurs polynomial de degré inférieur ou égal à $2^{k+1}+1$. On a la relation

$$
\|D(P)\|_{r_{k+1}} \leq \frac{2^{k+1}+1}{r_{k+1}}\|P\|_{r_{k+1}} ;
$$

(ii) si, de plus, $P$ est d'ordre supérieur ou égal à $2^{k}+2$, on a

$$
\|P\|_{r_{k}} \leq \gamma_{k}^{-2^{k}} \Longrightarrow\|P\|_{r_{k+1}} \leq \frac{1}{\left(2^{k}\right)^{2}} ;
$$

(iii) et, de plus,

$$
\|P\|_{r_{k}} \leq \gamma_{k}^{-2^{k}} \Longrightarrow\|D P\|_{r_{k+1}} \leq \frac{1}{2^{k-3}} .
$$

Preuve du lemme A.4. Pour (i) voir [8, p. 149]. Montrons (ii). Puisque le champ de vecteurs $P$ est d'ordre $2^{k}+2$, et comme $r_{k+1} \leq r_{k}$, on a

$$
\|P\|_{r_{k+1}} \leq\left(\frac{r_{k+1}}{r_{k}}\right)^{2^{k}+2}\|P\|_{r_{k}} \leq\left(\frac{r_{k+1}}{r_{k}}\right)^{2^{k}}\|P\|_{r_{k}} .
$$

Or, par définition de la suite $\left(r_{k}\right)_{k \geq 1}$, on a $r_{k+1} / r_{k}=\gamma_{k}\left(2^{k}\right)^{-\left(2 / 2^{k}\right)}$. De plus, on a $\left|\gamma_{k}\right| \leq 1$. D'où $\|P\|_{r_{k+1}} \leq\left(2^{k}\right)^{-2}$. On obtient (iii) à partir de (A.11) via $r_{k+1}>1 / 2$, et $\|D P\|_{r_{k+1}} \leq\left(\left(2^{k+1}+1\right) / r_{k+1}\right)\|P\|_{r_{k+1}}$. 
Les majorations suivantes sont conséquence directe de la définition de la norme $\|\cdot\|_{r}$. Soient $M$ un champ d'applications linéaires holomorphe, $r>0$ et $X$ un champ de vecteurs holomorphe. On a

$$
\|M(X)\|_{r} \leq n\|M\|_{r}\|X\|_{r} .
$$

Soient $A$ et $B$ deux champs de vecteurs holomorphes. On a

$$
\|A \wedge B\|_{r} \leq 2\|A\|_{r}\|B\|_{r}
$$

Preuve de la proposition 5.1(i). Soit $\Phi$ est un germe d'application de $\mathbb{C}^{n}$ dans un espace normé, on note, pour $y \in \mathbb{C}^{n}, D(\Phi)(y)$ la différentielle de $\Psi$ au point $y$. Soit $X$ un champ de vecteurs. On note $D(\Phi)(y) X(y)$ la différentielle de $\Psi$ en $y$, appliquée au vecteur $X(y)$. Pour $\Phi$ germe de difféomorphisme de $\left(\mathbb{C}^{n}, 0\right)$ dans lui même on a $D\left(\Phi^{-1}\right)(y) \Phi^{*}(X)(y)=$ $X\left(\Phi^{-1}(y)\right)$. Pour un bivecteur de la forme $A \wedge B$, cette relation devient

$$
(A \wedge B)\left(\Psi^{-1}(y)\right)=D\left(\Psi^{-1}\right)(y) \Psi^{*}(A)(y) \wedge D\left(\Psi^{-1}\right)(y) \Psi^{*}(B)(y) .
$$

Rappelons (cf. section 5) que la transformation $\Phi_{k}$, normalisant $\Pi$ du degré $2^{k}+1$ à celui de $2^{k+1}$, admet $\mathrm{Id}+U_{k}$ comme inverse. En appliquant (A.14), on obtient, avec $(\Phi)^{*}(A)=A^{\prime}$ et $(\Phi)^{*}(B)=B^{\prime}$, que

$$
\begin{aligned}
(A \wedge B)\left(\Phi_{k}^{-1}(y)\right) & =D\left(\Phi_{k}^{-1}\right)(y)\left(\Phi_{k}\right)^{*}(A)(y) \wedge D\left(\Phi_{k}^{-1}\right)(y)\left(\Phi_{k}\right)^{*}(B)(y) \\
& =\left(\operatorname{Id}+D U^{k}\right)(y) A^{\prime}(y) \wedge\left(\operatorname{Id}+D U^{k}\right)(y) B^{\prime}(y) .
\end{aligned}
$$

Comme $\Pi^{k+1}=\Pi^{k}+B_{0}^{k}$ on peut poser

$$
\begin{aligned}
\Pi^{k}+B_{*}^{k}+B_{0}^{k}+R^{k} & :=\Pi^{k}+R^{\prime k}:=\sum_{i=1}^{n} \partial_{i} \wedge\left(\Pi_{i}^{k}+R_{i}^{\prime k}\right) \\
\Pi^{k+1}+R^{\prime k+1} & :=\Pi^{k}+B_{0}^{k}+R^{\prime k+1} \\
& :=\sum_{i=1}^{n} \partial_{i} \wedge\left(\Pi_{i}^{k}+B_{0 i}^{k}+R_{i}^{\prime k+1}\right) .
\end{aligned}
$$

Majoration de $\left\|B_{*}^{k+1}+B_{0}^{k+1}+R^{k+1}\right\|_{r_{k+1}}$. Montrons d'abord que pour $k$ assez grand, (i) (a), (b) et (c) impliquent (i) (c') de la proposition 5.1, c'est-à-dire

$$
\left\|B_{*}^{k+1}+B_{0}^{k+1}+R^{k+1}\right\|_{r_{k+1}} \stackrel{(A .16)}{=}\left\|R^{\prime k+1}\right\|_{r_{k+1}} \leq 1 .
$$

Pour cela, d'après (A.17) et (A.18), il suffit de montrer que pour tout $1 \leq i \leq n$ on a $\left\|R_{i}^{\prime k+1}\right\|_{r_{k+1}} \leq 1 / n$. Le fait que $\Phi_{k}$ normalise $\Pi$ du degré $2^{k}+1$ à celui de $2^{k+1}$, s'écrit, avec les notations (A.16)-(A.18),

$$
\left(\Phi_{k}\right)^{*}\left(\Pi^{k}+R^{\prime k}\right)=\Pi^{k+1}+R^{\prime k+1}=\Pi^{k}+B_{0}^{k}+R^{\prime k+1} .
$$

Alors

$$
\left(\Pi^{k}+R^{\prime k}\right)\left(\Phi^{k}\right)^{-1}(y)=\sum_{i} \partial_{i} \wedge\left(\Pi_{i}^{k}\left(\Phi^{k}\right)^{-1}(y)+R_{i}^{\prime k}\left(\Phi^{k}\right)^{-1}(y)\right) .
$$


Par (A.20), $\left(\Pi^{k}+R^{\prime k}\right)\left(\Phi^{k}\right)^{-1}(y)=\left(\operatorname{Id}+D U^{k}\right)\left(\Pi^{k}+B_{0}^{k}+R^{\prime k+1}\right)(y)$. En substituant le premier membre de cette relation avec (A.21), et en développant le second membre avec (A.20), (A.17) et (A.15) (en posant $A^{\prime}=\partial_{i}$ et $B^{\prime}=\Pi_{i}^{k}+B_{0 i}^{k}+R_{i}^{\prime k+1}$ ),

$$
\begin{aligned}
\sum_{i} \partial_{i} & \wedge \Pi_{i}^{k}\left(\Phi^{k}\right)^{-1}(y)+R_{i}^{\prime k}\left(\Phi^{k}\right)^{-1}(y) \\
= & \sum_{i} \partial_{i} \wedge\left(\Pi_{i}^{k}(y)+B_{0 i}^{k}(y)+R_{i}^{\prime k+1}(y)\right) \\
& +\sum_{i} \partial_{i} \wedge\left(D U^{k}(y)\left(\Pi_{i}^{k}+B_{0 i}^{k}+R_{i}^{\prime k+1}\right)(y)\right) \\
& +\sum_{i}\left[D U^{k}(y)\left(\partial_{i}\right)\right] \wedge\left(\Pi_{i}^{k}+B_{0 i}^{k}+R_{i}^{\prime k+1}\right)(y) \\
& +\sum_{i}\left[D U^{k}(y)\left(\partial_{i}\right)\right] \wedge\left(D U^{k}(y)\left(\Pi_{i}^{k}+B_{0 i}^{k}+R_{i}^{\prime k+1}\right)(y)\right)
\end{aligned}
$$

Par hypothèse (cf. proposition 5.1(i)(c)) $\left\|B_{*}^{k}\right\|_{r_{k}} \leq\left\|B_{*}^{k}+B_{0}^{k}+R^{k}\right\|_{r_{k}} \leq 1$, et (cf. proposition 5.1(i)(a) et (i)(b) et (5.2)) $\left\|U_{k}\right\|_{r_{k}} \leq \gamma_{k}^{2^{k}}$. D'après le lemme A.4(iii), $\left\|D U_{k}\right\|_{r_{k+1}} \leq$ $1 / 2^{k-3}$. Avec (A.12) on a alors $\left\|D\left(U_{k}\right)(y) R_{i}^{\prime k+1}\right\|_{r_{k+1}} \leq\left(8 n / 2^{k}\right)\left\|R_{i}^{\prime k+1}\right\|_{r_{k+1}}$, d'où

$$
\left\|\sum_{i} \partial_{i} \wedge R_{i}^{\prime k+1}(y)+\sum_{i} \partial_{i} \wedge D U^{k}(y) R_{i}^{\prime k+1}(y)\right\|_{r_{k+1}} \geq\left(1-\frac{8 n}{2^{k}}\right)\left\|R_{i}^{\prime k+1}\right\|_{r_{k+1}} \text {. }
$$

D'après le lemme A.3, $\left\|D \Pi_{i}^{k}\left(y+t U^{k}(y)\right)\right\|_{r_{k+1}} \leq\left\|D \Pi_{i}^{k}(y)\right\|_{\left(2^{k}\right)^{-1 /\left(2^{k}\right)} r_{k}}$ pour $k$ assez grand. Alors avec le lemme A.4 et $\left(2^{k}\right)^{-1 / 2^{k}}<1$,

$$
\left\|D \Pi_{i}^{k}\left(y+t U^{k}(y)\right)\right\|_{r_{k+1}} \leq \frac{2^{k+1}+2}{\left(\left(2^{k}\right)^{-1 /\left(2^{k}\right)}\right) r_{k}}\left\|\Pi_{i}^{k}\right\|_{r_{k}} .
$$

Comme $\left\|U^{k}(y)\right\|_{r_{k}} \leq \gamma_{k}^{-2^{k}}$, on a $\left\|U^{k}(y)\right\|_{r_{k+1}} \leq 1 /\left(2^{k}\right)^{2}$, d'après le lemme A.4(ii). Avec (A.12) on en déduit

$$
\left\|\int_{0}^{1} D \Pi_{i}^{k}\left(y+t U^{k}(y)\right) \cdot U^{k}(y) d t\right\|_{r_{k+1}} \leq n \frac{2^{k+1}+2}{\left(\left(2^{k}\right)^{-1 /\left(2^{k}\right)}\right) r_{k}}\left\|\Pi_{i}^{k}\right\|_{r_{k}} \frac{1}{\left(2^{k}\right)^{2}},
$$

c'est-à-dire $\left(\left|\left(2^{k}\right)^{-1 /\left(2^{k}\right)} r_{k}\right|<1\right)$

$$
\left\|\int_{0}^{1} D \Pi_{i}^{k}\left(y+t U^{k}(y)\right) \cdot U^{k}(y) d t\right\|_{r_{k+1}} \leq \frac{4 n}{2^{k}}\left\|\Pi_{i}^{k}\right\|_{r_{k}} .
$$

Avec

$$
\left\|\Pi_{i}^{k}\left(\Phi^{k}\right)^{-1}(y)-\Pi_{i}^{k}(y)\right\|_{r_{k+1}}=\left\|\int_{0}^{1} D \Pi_{i}^{k}\left(y+t U^{k}(y)\right) \cdot U^{k}(y) d t\right\|_{r_{k+1}}
$$

il suit

$$
\left\|\Pi_{i}^{k}\left(\Phi^{k}\right)^{-1}(y)-\Pi_{i}^{k}(y)\right\|_{r_{k+1}} \leq \frac{4 n}{2^{k}}\left\|\Pi_{i}^{k}\right\|_{r_{k}} .
$$

Or, par (A.13), $\left\|\Pi_{i}^{k}(y)\right\|_{r_{k}} \leq 2\left\|\Pi^{k}(y)\right\|_{r_{k}}$ et $\left\|\Pi^{k}(y)-\Pi^{2}(y)\right\|_{r_{k}} \leq \eta$, avec $\eta$ tel que dans le théorème 3.5. Donc il existe $M>0$, indépendant de $k$, tel que $\left\|\Pi^{k}(y)\right\|_{r_{k}} \leq M$. Pour $k$ assez grand on a donc

$$
\left\|\Pi_{i}^{k}\left(\left(\Phi^{k}\right)^{-1}(y)\right)-\Pi_{i}^{k}(y)\right\|_{r_{k+1}} \leq \frac{4 n M}{2^{k}} .
$$


Comme $\left\|B_{0 i}^{k}\right\|_{r_{k}} \leq\left\|R_{i}^{\prime k}\right\|_{r_{k}}<1$, le lemme A.2 implique $\left\|B_{0 i}^{k}\right\|_{r_{k+1}} \leq 1 /\left(2^{k}\right)^{2}$. Comme $r_{k} \geq$ $r_{k+1},\left\|\Pi_{i}^{k}\right\|_{r_{k+1}} \leq M$. Comme $r_{k+1}>1 / 2,\left\|D U^{k}\right\|_{r_{k+1}} \leq 2^{k+3}\left\|U^{k}\right\|_{r_{k+1}}$ par le lemme A.4, et avec $\left\|U^{k}\right\|_{r_{k}} \leq \gamma^{-2^{k}},\left\|D U^{k}\right\|_{r_{k+1}} \leq 8 / 2^{k}$. Au total, avec (A.12), on en déduit

$$
\left\|D U^{k}(y)\left(\Pi_{i}^{k}+B_{0 i}^{k}\right)(y)\right\|_{r_{k+1}} \leq \frac{8 n}{2^{k}}\left(M+\frac{1}{\left(2^{k}\right)^{2}}\right) .
$$

Pour $k$ assez grand on a $\left\|R_{i}^{\prime k}\left(\Phi^{k}\right)^{-1}(y)\right\|_{r_{k+1}} \leq\left\|R_{i}^{\prime k}(y)\right\|_{\left(2^{k}\right)^{-1 /\left(2^{k}\right)} r_{k}}$ par le lemme A.3. Grâce au lemme A. 2 et à $\left\|R_{i}^{\prime k}(y)\right\|_{r_{k}} \leq 1$ il suit

$$
\left\|R_{i}^{\prime k}\left(\Phi^{k}\right)^{-1}(y)\right\|_{r_{k+1}} \leq \frac{1}{2^{k}}
$$

pour $k$ assez grand. Avec (A.24), $\left\|B_{0 i}^{k}\right\|_{r_{k+1}} \leq 1 /\left(2^{k}\right)^{2}$, (A.25) et (A.26), on a (pour $k$ assez grand)

$$
\begin{aligned}
& \| \sum_{i} \partial_{i} \wedge\left[\left(\Pi_{i}^{k}\left(\Phi^{k}\right)^{-1}(y)-\Pi_{i}^{k}(y)\right)+B_{0 i}^{k}(y)\right. \\
& \left.\quad+D U^{k}(y)\left(\Pi_{i}^{k}+B_{0 i}^{k}\right)(y)+R_{i}^{k}\left(\Phi^{k}\right)^{-1}(y)\right] \|_{r_{k+1}} \\
& \leq \frac{4 n M}{2^{k}}+\frac{1}{\left(2^{k}\right)^{2}}+\frac{8 n}{2^{k}}\left(M+\frac{1}{\left(2^{k}\right)^{2}}\right)+\frac{1}{2^{k}} .
\end{aligned}
$$

Avec $\left\|D U_{k}\right\|_{r_{k+1}} \leq 1 / 2^{k-3},\left\|\Pi_{i}^{k}\right\|_{r_{k+1}} \leq M$ et $\left\|B_{0 i}^{k}\right\|_{r_{k+1}} \leq 1 /\left(2^{k}\right)^{2}$ on obtient pour k assez grand (avec(A.12), (A.13))

$\left\|\sum_{i}\left[D U^{k}(y)\left(\partial_{i}\right)\right] \wedge\left(\Pi_{i}^{k}+B_{0 i}^{k}+R_{i}^{\prime k+1}\right)(y)\right\|_{r_{k+1}} \leq \frac{16 n}{2^{k}}\left(M+\frac{1}{\left(2^{k}\right)^{2}}+\left\|R_{i}^{\prime k+1}\right\|_{r_{k+1}}\right)$.

Pour les mêmes raisons,

$$
\begin{aligned}
& \left\|\sum_{i}\left[D U^{k}(y)\left(\partial_{i}\right)\right] \wedge\left(D U^{k}(y)\left(\Pi_{i}^{k}+B_{0 i}^{k}+R_{i}^{\prime k+1}\right)(y)\right)\right\|_{r_{k+1}} \\
& \quad \leq \frac{16 n}{2^{k}} \frac{4 n}{2^{k}}\left(M+\frac{1}{\left(2^{k}\right)^{2}}+\left\|R_{i}^{\prime k+1}\right\|_{r_{k+1}}\right) .
\end{aligned}
$$

D'où (pour $k$ assez grand),

$$
\begin{aligned}
& \left\|\sum_{i}\left[D U^{k}(y)\left(\partial_{i}\right)\right] \wedge\left[\left(\Pi_{i}^{k}+B_{0 i}^{k}+R_{i}^{\prime k+1}\right)(y)+\left(D U^{k}(y)\left(\Pi_{i}^{k}+B_{0 i}^{k}+R_{i}^{\prime k+1}\right)(y)\right)\right]\right\|_{r_{k+1}} \\
& \quad \leq \frac{17 n}{2^{k}}\left(M+\frac{1}{\left(2^{k}\right)^{2}}+\left\|R_{i}^{\prime k+1}\right\|_{r_{k+1}}\right) .
\end{aligned}
$$

De (A.22), (A.23), (A.27) et (A.29) on déduit pour $k$ assez grand

$$
\begin{aligned}
& \left(1-\frac{8 n}{2^{k}}-\frac{17 n}{2^{k}}\right)\left\|R_{i}^{\prime k+1}\right\|_{r_{k+1}} \\
& \leq \frac{4 n M}{2^{k}}+\frac{1}{\left(2^{k}\right)^{2}}+\frac{8 n}{2^{k}}\left(M+\frac{1}{\left(2^{k}\right)^{2}}\right)+\frac{1}{2^{k}}+\frac{17 n}{2^{k}}\left(M+\frac{1}{\left(2^{k}\right)^{2}}\right),
\end{aligned}
$$


d'où (A.19). Reste à montrer (i)(a') et (b'), soit $\left\|\Pi^{k+1}-\Pi^{0}\right\|_{r_{k+1}} \leq \eta-1 / 2^{k+1}$ et $\left\|D\left(\Pi^{k}-\Pi^{0}\right)\right\|_{r_{k+1}} \leq \eta-4 / 2^{k+1}$ pour $k$ assez grand. Avec $\Pi^{k+1}=\Pi^{k}+B_{0}^{k}$ on a

$$
\left\|\Pi^{k+1}-\Pi^{0}\right\|_{r_{k+1}} \leq \eta-\frac{4}{2^{k}}+\frac{1}{\left(2^{k}\right)^{2}}\left\|B_{0}^{k}\right\|_{r_{k}}
$$

par le lemme A.2 et $\left\|\Pi^{k}-\Pi^{0}\right\|_{r_{k}} \leq \eta-1 / 2^{k}$. Avec $\left\|B_{0}^{k}\right\|_{r_{k}} \leq 1$,

$$
\left\|\Pi^{k+1}-\Pi^{0}\right\|_{r_{k+1}} \leq \eta-\frac{4}{2^{k+1}}
$$

pour $k$ assez grand. De même,

$$
\left\|D\left(\Pi^{k+1}-\Pi^{0}\right)\right\|_{r_{k+1}} \leq \eta-\frac{4}{2^{k}}+\left\|D B_{0}^{k}\right\|_{r_{k+1}} \leq \eta-\frac{4}{2^{k}}+\frac{1}{2^{k-3}} \leq \eta-\frac{4}{2^{k+1}} .
$$

Preuve de la proposition 5.1(ii): Par la proposition 5.1(i), $\left\|B_{*}^{k}\right\|_{r_{k}} \leq 1$, d'où $\left\|U^{k}\right\|_{r_{k}} \leq$ $\gamma_{k}^{-1 / 2^{k}}$ et donc $\left\|U^{k}\right\|_{r_{k+1}} \leq 1 /\left(2^{k}\right)^{2}$. Par le lemme A.3 il suit $\left(\operatorname{Id}+U^{k}\right)\left(D_{r_{k+1}}\right) \subseteq$ $D_{\left(2^{k}\right)^{-1 /\left(2^{k}\right)} r_{k}}$.

\section{REFERENCES}

[1] V. I. Arnol'd. Mathematical Methods of Classical Mechanics, 2nd edn. (Graduate Texts in Mathematics, 60). Springer, Berlin, 1989.

[2] A. D. Bruno. The analytical form of differential equations. Trans. Moscow Soc. 25 (1971), 131-288; 26 (1972), 199-239.

[3] J. Conn. Normal forms for analytic Poisson structures. Ann. of Math. (2) 119 (1984), 577-601.

[4] J. Conn. Correction to "Normal forms for analytic Poisson structures". Ann. of Math. (2) 121 (1985), 433-436.

[5] J. P. Dufour and A. Haraki. Rotationnels et structures de Poisson quadratiques. C. R. Acad. Sci. Paris Sér. I 312 (1991), 137-140.

[6] J. P. Dufour and A. Wade. Formes normales de structures de Poisson ayant un 1-jet nul en un point. J. Geom. Phys. 26 (1998), 79-96.

[7] J. P. Dufour and N. T. Zung. Poisson Structures and their Normal Forms (Progress in Mathematics, 242). Birkhäuser, Basel, 2005.

[8] L. Stolovitch. Singular complete integrability. Publ. Math. Inst. Hautes. Études. Sci. 91 (2000), 133-210.

[9] L. Stolovitch. Sur les structures de Poisson singulières. Ergod. Th. \& Dynam. Sys. 24(5) (2004), 1833-1863.

[10] A. Weinstein. The local structure of Poisson manifolds. J. Differential Geom. 18 (1983), 523-557.

[11] A. Wade. Normalisation de structures de Poisson. PhD Thesis, Université Montpellier, 1996. 